\title{
Evolution-equation approach to seismic image, and data, continuation ${ }^{1}$
}

\author{
Anton A. Duchkov ${ }^{\mathrm{a}, *}$, \\ Maarten V. de Hoop ${ }^{\mathrm{a}}$ and Antônio Sá Barreto ${ }^{\mathrm{a}, 2}$ \\ ${ }^{a}$ Center for Computational and Applied Mathematics, Purdue University, $150 \mathrm{~N}$. \\ University Street, West Lafayette IN 47907, USA \\ In memory of our dear friend Sergey Vasilievich Goldin
}

\begin{abstract}
In reflection seismology one places point sources and point receivers on the earth's surface, forming an acquisition geometry. Each source generates acoustic waves in the subsurface, that are reflected where the medium properties vary discontinuously. The reflections that can be observed at the receivers are used to image these discontinuities or reflectors assuming a background medium. We analyze methods to circumvent the repeated imaging of reflectors under varying background media, or the repeated modelling of reflections under varying acquisition geometries. These methods involve the introduction of the notion of seismic continuation. Here, we develop the foundation of, and a comprehensive framework for seismic continuation while extending earlier approaches to allow for the formation of caustics. Traditionally, seismic continuation has been viewed from a geometrical (ray asymptotic) point of view; here, we introduce the notion of wave-equation continuation through the appearance of evolution equations.
\end{abstract}

Key words: velocity continuation, Fourier integral operators, evolution systems, global Hamiltonians

\footnotetext{
Email address: aduchkov@purdue.edu (Anton A. Duchkov).

URL: http: / / www . math. purdue. edu/ aduchkov/site (Anton A. Duchkov).

1 This research was supported in part under NSF CMG grant EAR-0417891.

2 Supported under NSF grant DMS-0500788.
} 


\section{Introduction}

In reflection seismology one places point sources and point receivers on or near the earth's surface. Each source generates acoustic waves in the subsurface, that are reflected where the medium properties vary discontinuously. (We allow both passive sources, in global earth applications, and active sources, in exploration seismology.) The recorded reflections that can be observed at the receivers are used to image these discontinuities or reflectors.

Seismic reflection data, in the single scattering or Born approximation, are commonly modelled by an integral operator mapping a medium contrast (containing reflectors), given a background medium (velocity model), to a wavefield (containing reflections). Imaging of seismic reflection data is then described by the adjoint of this integral operator with a given background medium. In exploration seismology, the process of imaging is also referred to as migration, while the process of modelling data from an image is referred to as demigration. In applications, however, the background medium may not be accurately known, and hence it becomes desirable to develop a family of modelling and imaging operators for a set of background media. Also, the data may have been acquired for one particular acquisition geometry, while it can become desirable to generate the data for different geometries, requiring the development of an associated family of imaging and modelling operators. The latter can be viewed as a method of data regularization.

In present day applications, the volume of data can be massive, whence it becomes advantageous to circumvent the repeated imaging or migration under varying background media or the repeated modelling or demigration under varying acquisition geometries. This leads to the introduction of the notion of seismic continuation: The continuation of an image following a path of background media without remigrating the data, or the continuation of data following a path of acquisition geometries without demigrating an image. The applications encompass the exploration of discontinuities in Earth's interior.

The notion of seismic continuation has been around for many years. Fomel introduced the concept of data continuation in source-receiver offset in [1] ${ }^{3}$ and the concept of image continuation with velocity in [3]; this continuation assumed constant background media. Data continuation allows, under certain conditions, to obtain zero-offset (coinciding sources and receivers) data can be obtained from finite-offset data, as in the so-called data transformation to zero offset (TZO) obtained after dip moveout (DMO) (see, for example, also [4,5]). An approach similar to image continuation is residual migration (see [6-8]). The concept of image continuation in varying background media was further developed by Hubral, Tygel and Schleicher $[9,10]$, Fomel [11], Iversen [12,13] and Adler [14]. In the process of image continuation, one can also track the impulse response of the imaging operator; in connection with this, Iversen [15] intro-

$\overline{3}$ in [2] the authors erroneously attributed the equation generating this continuation, and the underlying geometrical construction, to S.V. Goldin. 
duced the notion of isochron rays. Residual velocity DMO introduced by Alkhalifah and De Hoop [16,17] yields TZO in conjunction with continuation following a path of anisotropic velocity models and is reminiscent of both these concepts. Continuation in background velocity can be exploited in developing a method for determining it. For the case of image continuation, consistent with terminology from the seismic literature, we refer to such a method as 'continuation-based' migration velocity analysis. This idea was explored by Liu and Bleistein [18] and Meng and Bleistein [19,20].

The above mentioned, sometimes seemingly different concepts, were developed either for constant background media or under the condition of absence of caustics. In a more general setting the theory of continuation and a question about its realization by GRT integral operators or partial differential equations was considered by Goldin [21-23] in the context of contact geometry. His work has inspired us to develop the foundation of, and a common, comprehensive, framework for seismic continuation while extending the earlier approaches to allow for the formation of caustics. Furthermore, we establish that the propagation of singularities by continuation can always be expressed in terms of a canonical transformation, while we address the question whether continuation can be described by a hyperbolic evolution system. Indeed, we introduce necessary and sufficient conditions for the notion of continuation to be well-defined, and show the existence of an evolution equation that dynamically generates the continuation. The principal symbol of this equation defines a (global) Hamiltonian, the flow of which defines continuation bicharacteristics and describes the continuation of singularities.

Our main analytical tools are taken from microlocal analysis, see e.g. [24-27]. Modelling and imaging of seismic reflection data can be mathematically described in terms of Fourier integral operators (FIOs) [28-32]. The basic properties of FIOs are summarized in Section 2. The class of FIOs the canonical relations of which are graphs, and which are invertible, forms the key building block of seismic continuation theory. For the modelling (and imaging) operators, in the presence of caustics, to be contained in this class, these operators need to be extended (Stolk and De Hoop [32,33]).

In Section 3 we develop a theory of continuation. For this we introduce smooth one-parameter families of FIOs in the above mentioned class. Then we prove that such a family can be identified as solution operators to hyperbolic pseudodifferential evolution equations. (The parameter becomes an evolution parameter - it imitates the time in wave propagation.) Thus, continuation operators propagate singularities along the bicharacteristics that solve a Hamilton system, in which the Hamiltonian is derived from the principal symbol of the evolution operator. (The bicharacteristics are also curves in phase space determined by the canonical transformation.) Continuation operators form a class of invertible FIOs of order 0 , the canonical relations of which are graphs. This class has a principal fiber bundle, and Lie group, structure. The base space is formed by the canonical transformations, which reflects the central role that the geometry plays in seismic continuation. Continuation can then be formalized as a curve in a section of this principal fiber bundle. Canonical transformations are identifiable with contact transforma- 
tions, which have been used in an alternative description of propagation of singularities through the notion of contact elements (Goldin [22]).

In Section 4 we present three interconnected approaches to the construction continuation. In Section 4.1, we consider the approach based on the composition of canonical relations and amplitudes. In Section 4.2 we establish the explicit relation between the phase function in the kernel representation of the original FIO, the generating function corresponding with its canonical graph, and the pseudodifferential operator symbol appearing in the above mentioned evolution equation. This relation can be used as a construction in applications. The Hamiltonian that generates the continuation bicharacteristics is expressed in terms of this generating function. The continuation bicharacteristics can also be constructed directly from the evolution of fronts using the phase function. Essentially, the geometry associated to seismic continuation has been inferred from the geometry of migration operators. In Section 4.3 we show how to obtain an alternative geometrical description of the continuation providing continuation bicharacteristics without explicit use of the Hamiltonian. This is done by composing the phases in the kernel representations of the FIOs making up the continuation operator and differentiating with respect to parameter value. In applications, often, a vector field tangent to the Hamiltonian flow can be directly constructed; using Poincaré's lemma, a global Hamiltonian can be obtained from such a vector field.

In Sections 5 and 6 we conclude with showing examples of continuation in reflection seismology. The examples in Section 5 are derived from imaging with the generalized Radon transform (encompassing Kirchhoff migration). We give explicit expressions for phase functions and generating functions associated to the kernels of the relevant FIOs. In Section 5.3, we demonstrate how earlier concepts are contained in our theory. In Section 5.1 we discuss the original presentation (derived from the phase function) of image continuation under common-offset Kirchhoff migration in the absence of caustics, and specialize to constant background media. One of the motivations for developing the theory presented in this paper was indeed to establish the connection between continuation as a composition of migration with demigration and the construction of 'velocity rays' to describe the propagation of singularities under continuation (Section 5.3); a second motivation was to bring the system of ordinary differential equations for continuation bicharacteristics in Hamilton form (Sections 5.1 and 5.2). A third motivation was to establish the importance of canonical transformations (preserving the symplectic form) generating continuation operators.

In Section 6, we discuss the notion of image gathers and their velocity continuation in the presence of caustics. We show an example, revealing the potential of the comprehensive theory presented here (Section 4). Velocity continuation of image gathers can directly be exploited in reflection tomography, the problem of determining the background velocity, see [34,35,32,36,37]. 


\section{Representations of 'migration' and 'demigration' operators}

We formulate modelling and imaging of seismic reflection data within the general framework of linear integral operators. Let $y$ denote a point in an acquisition manifold $Y$ on which data $u$ are defined. Let $x$ denote a point in the subsurface manifold $X$ on which a contrast $v$ or an image $w$ is defined. We let $n_{X}=\operatorname{dim} X$ and $n_{Y}=\operatorname{dim} Y$; naturally, $n_{Y} \geq n_{X}$. Typically, $y$ consists of a combination of source and receiver points contained in $\partial X$, and time. We consider the operator pair $F, F^{*}$, where $F^{*}$ is the adjoint of $F$, that is $\langle u, F v\rangle_{Y}=\left\langle F^{*} u, v\right\rangle_{X}$. For any data $u$, there exists a $v \in \mathcal{E}^{\prime}(X)$ such that

$$
u=F v
$$

In general, $u \in \mathcal{D}^{\prime}(Y)$. Moreover, $w$ in

$$
w=F^{*} u
$$

is identified as the image.

We assume that $F$ is a Fourier integral operator (FIO) - this assumption is commonly satisfied in seismic data applications [28-32]. Then $F^{*}$ is an FIO as well. The action of $F$, microlocally, can be written in the form

$$
\begin{aligned}
(F v)(y) & =\int A(y, x) v(x) \mathrm{d} x \\
A(y, x) & =\int_{\mathbb{R}^{N}} a(y, x, \theta) \exp [\mathrm{i} \phi(y, x, \theta)] \mathrm{d} \theta
\end{aligned}
$$

in which $\theta=\left(\theta_{1}, \ldots, \theta_{N}\right)$ are so-called phase variables. Here, $\phi$ is a phase function: $\phi$ is realvalued, $\phi \in C^{\infty}\left(Y \times X \times\left(\mathbb{R}^{N} \backslash 0\right)\right)$, $\phi$ is positive-homogeneous of degree one in $\theta$, and $\phi$ does not have critical points for $\theta \neq 0$, that is, $\partial_{(y, x)} \partial_{\theta} \phi(y, x, \theta) \neq 0$ for $(y, x) \in Y \times X$ and $\theta \in \mathbb{R}^{N} \backslash 0$.

Furthermore, $a$ is an amplitude of order $m$, that is $a \in S^{m}\left(Y \times X, \mathbb{R}^{N}\right)$, which has the property: To every compact subset $\mathcal{K} \subset Y \times X$ and multi-indices $\alpha, \beta$ there is a constant $C_{\alpha, \beta}(\mathcal{K})$ such that

$$
\begin{gathered}
\left|\partial_{\theta}^{\alpha} \partial_{(y, x)}^{\beta} a(y, x, \theta)\right| \leq C_{\alpha, \beta}(\mathcal{K})\langle\theta\rangle^{m-\rho|\alpha|+\delta|\beta|}, \quad\langle\theta\rangle=\left(1+\|\theta\|^{2}\right)^{1 / 2}, \\
\quad \text { for all }(y, x) \in \mathcal{K} \text { and } \theta \in \mathbb{R}^{N} \backslash 0 .
\end{gathered}
$$

(With these estimates, and $\phi$ being a phase function, the integral representation for $A(y, x)$ in (4) can be regularized.) We restrict our analysis to the case $\rho=1, \delta=0$; amplitudes of order $m$ 
of this type define the class of FIOs $I^{M(m)}(X)$ (details on the order $M(m)$ may be found in [24, p. 41]). The operator $F$ extends to a continuous linear map $F: \mathcal{E}^{\prime}(X) \rightarrow \mathcal{D}^{\prime}(Y)$. The operator $F$ propagates singularities. Microlocally, this is determined by the phase function $\phi$, and can be understood as follows. The stationary point set of the phase function is given by

$$
S_{\phi}=\left\{(y, x, \theta) \mid \partial_{\theta} \phi(y, x, \theta)=0\right\} .
$$

The phase function will be assumed to be non-degenerate, that is, the rank of the Hessian matrix,

$$
\left.\left(\mathrm{d}_{(y, x, \theta)} \frac{\partial \phi}{\partial \theta}\right) \text { is maximal (that is, } N\right) \text {. }
$$

Then $S_{\phi}$ is a $\left(n_{Y}+n_{X}\right)$ - dimensional submanifold of $Y \times X \times\left(\mathbb{R}^{N} \backslash 0\right)$. Moreover, $S_{\phi}$ is a conic subset of $Y \times X \times\left(\mathbb{R}^{N} \backslash 0\right)$, i.e., if $\left(y_{0}, x_{0}, \theta_{0}\right) \in S_{\phi}$ then $\left(y_{0}, x_{0}, t \theta_{0}\right) \in S_{\phi}$ for any $t>0$.

In view of the homogeneity of $\phi$, we have $\phi=\theta \cdot \partial_{\theta} \phi$ (Euler's theorem), so that $\phi(y, x, \theta)=0$ if $(y, x, \theta) \in S_{\phi}$. Let $T^{*} Y \backslash 0$ denote the acquisition phase space and $T^{*} X \backslash 0$ denote the subsurface phase space. The stationary point set can be embedded in $T^{*} Y \backslash 0 \times T^{*} X \backslash 0$ :

$$
\begin{aligned}
S_{\phi} \rightarrow \Lambda, \quad(y, x, \theta) \rightarrow\left(y, \partial_{y} \phi ; x,-\partial_{x} \phi\right) \quad \text { is an immersion, } \\
\Lambda=\left\{\left(y, \partial_{y} \phi ; x,-\partial_{x} \phi\right) \mid \partial_{\theta} \phi=0\right\} .
\end{aligned}
$$

$\Lambda$ is (locally) a conic Lagrangian submanifold of $T^{*}(Y \times X) \backslash 0$, and is called the canonical relation of operator $F$; we sometimes write $\Lambda=\Lambda^{F}$ to indicate its association to $F$. It is immediate that $\Lambda^{F^{*}}=\left(\Lambda^{F}\right)^{*}=\left\{(x, \xi ; y, \eta) \mid(y, \eta ; x, \xi) \in \Lambda^{F}\right\}$. The canonical relation describes the propagation of singularities in (1): if WF denotes the wavefront set of a distribution,

$$
\begin{aligned}
& \mathrm{WF}(u) \subseteq \Lambda^{F} \circ \mathrm{WF}(w) \\
& \quad=\left\{(y, \eta) \mid(y, \eta ; x, \xi) \in \Lambda^{F} \text { and }(x, \xi) \in \mathrm{WF}(w) \text { for some }(x, \xi) \in T^{*} X \backslash 0\right\}, u=F w .
\end{aligned}
$$

Identifying reflections in $\mathrm{WF}(u)$ ( $\eta$ defines 'slopes') and reflectors in $\mathrm{WF}(w)$ or $\mathrm{WF}(v)(\xi$ defines 'dip'), following seismic terminology, we refer to $F^{*}$ as 'migration'; if $F$ acts on an image $w$, we speak of $F$ as 'demigration' instead of modelling.

The kernel $A$ in (4) is a Lagrangian distribution. Its singular support is also determined by the phase function $\phi$ : Let $\pi: Y \times X \times\left(\mathbb{R}^{N} \backslash 0\right) \rightarrow Y \times X$ denote the natural projection, then $\operatorname{sing} \operatorname{supp} A \subset \pi S_{\phi}$. Viewing $\operatorname{sing} \operatorname{supp} A$ at a fixed $x=x_{0}$ yields the physical notion of a front: $\mathcal{W}\left(x_{0}\right)=\left\{y \in Y \mid\left(y, x_{0}\right) \in \pi S_{\phi}\right\}$. In the reflection seismology literature, one refers to such a front as the (geometrical) 'impulse response' and 'special surfaces', see Goldin [22]. In case of modelling or demigration, the fronts are also called 'diffraction surfaces', while in the case of imaging these fronts are also called 'isochrons'. 
In general, $\Lambda$ admits local coordinates $\left(y_{I^{\prime}}, \eta_{J^{\prime}}, x_{I}, \xi_{J}\right)$ with $\left(I^{\prime} \cup I\right) \cup\left(J^{\prime} \cup J\right)=\left\{1,2, \ldots, n_{Y}+\right.$ $\left.n_{X}\right\}$ together with the existence of a generating function $S=S\left(y_{I^{\prime}}, \eta_{J^{\prime}}, x_{I}, \xi_{J}\right)$ such that

$$
\begin{aligned}
& x_{J}=\frac{\partial S}{\partial \xi_{J}}, \xi_{I}=-\frac{\partial S}{\partial x_{I}}, \\
& y_{J^{\prime}}=\frac{\partial S}{\partial \eta_{J^{\prime}}}, \eta_{I^{\prime}}=\frac{\partial S}{\partial y_{I^{\prime}}}
\end{aligned}
$$

[26, Thm. 21.2.18]. Then the phase variables in (4) can be locally chosen to be $\theta=\left(\eta_{J^{\prime}}, \xi_{J}\right)$, whence the phase function attains the form

$$
\phi\left(y, x, \eta_{J^{\prime}}, \xi_{J}\right)=S\left(y_{I^{\prime}}, \eta_{J^{\prime}}, x_{I}, \xi_{J}\right)-\left\langle\eta_{J^{\prime}}, y_{J^{\prime}}\right\rangle-\left\langle\xi_{J}, x_{J}\right\rangle
$$

Let $F_{1}$ and $F_{2}$ both be FIOs. $F_{2}$ maps functions on $X$ to functions on $Y$, and $F_{1}$ maps functions on $Y$ to functions on $Z$. The composition $F_{1} F_{2}$ is well defined if the intersection of $\Lambda^{F_{1}} \times \Lambda^{F_{2}}$ with $T^{*} Z \backslash 0 \times \operatorname{diag}\left(T^{*} Y \backslash 0\right) \times T^{*} X \backslash 0$ is transversal [25, Ch. VIII, p.464]. The canonical relation of the composition is given by $\Lambda^{F_{1}} \circ \Lambda^{F_{2}}$, following

$$
\begin{aligned}
\Lambda^{F_{1}} \times \Lambda^{F_{2}} & \cap T^{*} Z \backslash 0 \times \operatorname{diag}\left(T^{*} Y \backslash 0\right) \times T^{*} X \backslash 0 \\
& \downarrow \text { projection } \\
\Lambda^{F_{1}} & \circ \Lambda^{F_{2}} \subset T^{*} Z \backslash 0 \times T^{*} X \backslash 0
\end{aligned}
$$

We now develop the necessary preparation of continuation theory, which leads to a certain class of allowable FIOs. To begin with, we need to assume that $n_{Y}=n_{X}$.

Graph assumption. The canonical relation (cf. (8)) is a graph, that is, there exists a transformation $\Sigma: T^{*} X \rightarrow T^{*} Y$ such that

$$
\Lambda=\{(\Sigma(x, \xi) ; x, \xi)\}
$$

The transformation $\Sigma$ will be a canonical transformation, that is, it preserves the symplectic form. (If $\Sigma$ is the identity, $\Lambda^{F} \subset \operatorname{diag} T^{*} X \backslash 0$, the associated operator will simply be pseudodifferential.)

Subject to the graph assumption, the kernel of an FIO $F$ admits a representation (cf. (4), (9) with $\left|I^{\prime}\right|=n_{Y},|J|=n_{X}$, i.e. $\theta=\xi$ )

$$
\begin{aligned}
A(y, x) & =\int a(y, \xi) \exp [\mathrm{i} \phi(y, x, \xi)] \mathrm{d} \xi, \\
\phi(y, x, \xi) & =S(y, \xi)-\langle\xi, x\rangle,
\end{aligned}
$$


[27, Proposition 25.3.3] where $S$ is homogeneous of degree 1 in $\xi$; this representation is close to the one for a pseudodifferential operator kernel (for which $S(y, \xi)=\langle\xi, y\rangle$, so that $\phi(y, x, \xi)=$ $\langle\xi, y-x\rangle$ ). In (12) we have reduced the amplitude $a(y, x, \xi)$ to $a(y, \xi)$ by standard methods. Up to principal parts, $a_{0}(y, \xi)=a\left(y, \partial_{\xi} S, \xi\right)$. By an iteration argument [27, p.27] the amplitude $a(y, \xi)$ is obtained, leading to a kernel equivalent to the original operator kernel modulo $C^{\infty}$.

The principal symbol of $F$ with an integral kernel (12) is defined to be

$$
\sigma_{0}(F)(y, \xi)=a\left(y, \partial_{\xi} S(y, \xi), \xi\right)\left|\operatorname{det} \partial_{y} \partial_{\xi} S(y, \xi)\right|^{-\frac{1}{2}} .
$$

The canonical relation attains the form (cf. (8)-(9))

$$
\Lambda=\left\{\left(y, \partial_{y} S ; \partial_{\xi} S, \xi\right)\right\}
$$

(Indeed, a canonical transformation (cf. (11)) provides $S$, which generates a phase function as in (13)). In conjunction with this, the matrix $\partial_{\xi} \partial_{y} S$ is non-singular. Naturally, $(x, \xi)$ form coordinates on $\Lambda$ as well. How to change between representations of the type (4) and (12)-(13), with different phase variables, is discussed in Appendix A.

\section{Continuation theory}

We begin with introducing and describing one-parameter families of operators parameterized by $\alpha$, where $\alpha$ is taking values in an interval, $I=\left[\alpha_{1}, \alpha_{2}\right] \subset \mathbb{R}$.

DEFINITION 1 For each $\alpha \in I$, let $F(\alpha): \mathcal{E}^{\prime}(X) \longrightarrow \mathcal{D}^{\prime}(Y)$ be a properly supported FIO of order $m$ associated to the graph of a canonical transformation $\Sigma_{\alpha}$. We say that $F(\alpha)$ is a smooth, or $C^{\infty}$, family of FIOs if the following condition holds:

$$
\text { for all } f \in C_{0}^{\infty}(X), \quad F(\alpha) f \in C^{\infty}(I \times Y) \text {. }
$$

We define the operator $\partial_{\alpha} F(\alpha)$ as

$$
\left(\partial_{\alpha} F(\alpha)\right) f=\partial_{\alpha}(F(\alpha) f)
$$

We assume that

$$
\partial_{\alpha} F(\alpha) \text { is an FIO of order } m+1 \text {, associated to the graph of } \Sigma_{\alpha} .
$$

In terms of oscillatory integral representations, one characterizes a $C^{\infty}$ family of FIOs as follows. Let $F(\alpha), \alpha \in I$, be such a $C^{\infty}$ one-parameter family of FIOs of order zero associated 
to the graphs of canonical transformations $\Sigma_{\alpha}$ as above. For each $\alpha_{0} \in I$ and $\left(x_{0}, \xi_{0}, y_{0}, \eta_{0}\right)$ on the graph of $\Sigma_{\alpha_{0}}$ there exists a neighborhood $E \subset I$ of $\alpha_{0}$ and conic neighborhoods, $\Gamma$, of $\left(x_{0}, \xi_{0}, y_{0}, \eta_{0}\right)$ such that the Schwartz kernel, $A=A_{F(\alpha)}$, of $F(\alpha)$ is, microlocally, given by (12)-(13) with $a=a(\alpha, y, \xi)$ and $\phi=\phi(\alpha, y, x, \xi)$. Indeed, because

$$
\partial_{\alpha} A_{F(\alpha)}(y, x)=\int_{\mathbb{R}^{N}} \exp [\mathrm{i} \phi(\alpha, y, x, \theta)]\left(\mathrm{i}\left(\partial_{\alpha} \phi\right) a(\alpha, y, x, \theta)+\partial_{\alpha} a(\alpha, y, x, \theta)\right) \mathrm{d} \theta,
$$

it follows that $\partial_{\alpha} F(\alpha)$ is an FIO of order one associated to the graph of $\Sigma_{\alpha}$.

THEOREM 2 Let $F(\alpha), \alpha \in I$ be a $C^{\infty}$ family of properly supported invertible FIOs of order $m$. Then there exists a $C^{\infty}$ family of pseudodifferential operators, $P(\alpha)=P\left(\alpha ; y, D_{y}\right) \in$ $\Psi^{1}(Y)$, such that

$$
\left(\partial_{\alpha}-\mathrm{i} P(\alpha)\right) F(\alpha)=0 .
$$

Proof: For a smooth family of invertible FIOs $F(\alpha)$ we know that $\partial_{\alpha} F(\alpha)$ is an FIO of order $m+1$ associated to the graph of $\Sigma_{\alpha}$. Then by the calculus of FIOs, the operator

$$
\mathrm{i} P(\alpha)=\partial_{\alpha} F(\alpha) F(\alpha)^{-1} \in \Psi^{1}(Y) .
$$

Then it is immediate that (20) is satisfied.

Conversely,

THEOREM 3 Let $\alpha_{0} \in I$ and suppose that $F\left(\alpha_{0}\right)$ is a properly supported invertible FIO of order 0 , associated to the canonical transformation, $\Sigma_{\alpha_{0}}$. Let $P(\alpha) \in \Psi^{1}(Y)$ be a $C^{\infty}$ family of pseudodifferential operators with principal symbols $p_{1}(\alpha)$. Let $F(\alpha)$ be a family of operators satisfying equation (20). Then $F(\alpha)$ is a $C^{\infty}$ family of FIOs associated to $\Sigma_{\alpha}$, which is the canonical transformation whose graph is obtained from $\Sigma_{\alpha_{0}}$ by the flow of $H_{p_{1}(\alpha)}$. That is,

$$
\Sigma_{\alpha}=\exp \left(\left(\alpha-\alpha_{0}\right) H_{p_{1}(\alpha)}\right) \circ \Sigma_{\alpha_{0}}
$$

The proof of this result coincides with the proof of [38, Theorem 1.1].

DEFINITION 4 We reserve the notation $\mathcal{C}$ for the class of properly supported invertible FIOs of order 0 that satisfy the graph assumption (then the $\Sigma$ are diffeomorphisms).

The class $\mathcal{C}$ is an infinite-dimensional manifold with the structure of a principal fibre bundle: The base manifold consists of all canonical transformations, the fibres are isomorphic to the algebra of pseudodifferential operators of order 0 , while the structure group is that same algebra of pseudodifferential operators. In fact, the class $\mathcal{C}$ admits an infinite-dimensional Lie group structure $[39,40]$. Due to the group property, operators $F_{1}$ and $F_{2}$ in $\mathcal{C}$ compose transversally, 
and $F_{1} F_{2}$ is an FIO in $\mathcal{C}$ again. This structure is implicit in the original treatment and characterization of seismic data processing by Goldin.

Motivated by Theorems 2 and 3, we introduce the notion of continuation operator:

$$
C_{\left(\alpha, \alpha_{0}\right)}=F(\alpha) F\left(\alpha_{0}\right)^{-1}
$$

where the $F(\alpha)$ are properly supported invertible FIOs for $\alpha \in I$. Thus, for fixed $\alpha_{0}, C_{\left(\alpha, \alpha_{0}\right)}$ defines a one-parameter family of FIOs in $\mathcal{C}$ (cf. Definition 4), satisfying the semi-group property,

$$
C_{\left(\alpha, \alpha_{0}\right)}=C_{\left(\alpha, \alpha^{\prime}\right)} C_{\left(\alpha^{\prime}, \alpha_{0}\right)}, \quad \alpha_{0} \leq \alpha^{\prime} \leq \alpha
$$

Evolution equation. Theorem 2 guarantees that $C_{\left(\alpha, \alpha_{0}\right)}$ is the solution operator to a Cauchy problem (for a hyperbolic pseudodifferential evolution equation of the type (20)):

$$
\left(\partial_{\alpha}-\mathrm{i} P(\alpha)\right) w(\alpha, y)=0,\left.\quad w(\alpha, y)\right|_{\alpha=\alpha_{0}}=w\left(\alpha_{0}, y\right) .
$$

Thus $C_{\left(\alpha, \alpha_{0}\right)}$ can be called a propagator.

Continuation bicharacteristics. The canonical relation of $C_{\left(\alpha, \alpha_{0}\right)}$ is denoted by $\Lambda_{\left(\alpha, \alpha_{0}\right)}$; we denote the canonical transformation that generates $\Lambda_{\left(\alpha, \alpha_{0}\right)} \subset T^{*} Y \backslash 0 \times T^{*} Y \backslash 0$ by $\Sigma_{\left(\alpha, \alpha_{0}\right)}$, with $\Sigma_{\left(\alpha_{0}, \alpha_{0}\right)}=$ Id. The propagation of singularities by $C_{\left(\alpha, \alpha_{0}\right)}$ is then described by

$$
\Lambda_{\left(\alpha, \alpha_{0}\right)} \circ \mathrm{WF}\left(u\left(\alpha_{0}, .\right)\right)=\left\{\Sigma_{\left(\alpha, \alpha_{0}\right)}\left(y_{0}, \eta_{0}\right) \mid\left(y_{0}, \eta_{0}\right) \in \mathrm{WF}\left(u\left(\alpha_{0}, .\right)\right)\right\},
$$

through curves on $T^{*} Y \backslash 0$.

On the other hand, evolution equation (20) propagates singularities in accordance with the Hamilton flow with Hamiltonian

$$
\mathcal{H}\left(\alpha, y, \eta_{\alpha}, \eta\right)=\eta_{\alpha}-p_{1}(\alpha, y, \eta)
$$

where $p_{1}$ denotes the principal symbol of $P ; p_{1}$ is homogeneous of degree 1 in $\eta$. The Hamilton system is

$$
\begin{aligned}
\frac{\mathrm{d} y}{\mathrm{~d} \alpha}=\partial_{\eta} \mathcal{H}=-\partial_{\eta} p_{1}, \frac{\mathrm{d} \eta}{\mathrm{d} \alpha} & =-\partial_{y} \mathcal{H}=\partial_{y} p_{1}, \\
\frac{\mathrm{d} \eta_{\alpha}}{\mathrm{d} \alpha} & =-\partial_{\alpha} \mathcal{H}=\partial_{\alpha} p_{1},
\end{aligned}
$$

supplemented with initial conditions $y\left(\alpha_{0}\right)=y_{0}, \eta\left(\alpha_{0}\right)=\eta_{0}$, and $\eta_{\alpha}\left(\alpha_{0}\right)=\eta_{\alpha 0}$. In general, the Hamiltonian will be anisotropic even when one restricts to isotropic background media. In 
view of the homogeneity in $\eta$ we have the usual relation between (anisotropic) group velocity and slowness vectors, $\eta \cdot \frac{\mathrm{d} y}{\mathrm{~d} \alpha}=-\eta_{\alpha}$.

Equation (25) does not depend on $\eta_{\alpha}$ and thus may be solved independently. Solutions to (25) describe the canonical transformation generating $C_{\left(\alpha, \alpha_{0}\right)}$ for each $\alpha \in I$ fixed (cf. [41, section 5.3], see also [27, Theorem 21.3.4]). We refer to the solutions $y\left(\alpha, y_{0}, \eta_{0}\right), \eta\left(\alpha, y_{0}, \eta_{0}\right)$, determining $\Sigma_{\left(\alpha, \alpha_{0}\right)}$, that is, $\Sigma_{\left(\alpha, \alpha_{0}\right)}\left(y_{0}, \eta_{0}\right)=\left(y\left(\alpha, y_{0}, \eta_{0}\right), \eta\left(\alpha, y_{0}, \eta_{0}\right)\right)$, as the continuation bicharacteristics.

Remark. With $F$ being invertible, the graph assumption also holds for $F^{*}$; thus, $\Lambda^{*}$, and $\Lambda$, admit coordinates $(x, \eta)$. To suppress the detailed account of amplitudes, without loss of generality, we can assume that the FIOs are unitary, that is $F^{*}=F^{-1}$. Indeed, the normal operator $N=F^{*} F$ is pseudodifferential, and, by standard arguments, the polar decomposition $F=\widetilde{F} N^{1 / 2}$ provides $\widetilde{F}$ with $\widetilde{F}^{*}=\widetilde{F}^{-1}$; thus our further analysis applies to $\widetilde{F}$. Moreover, the above consideration can be generalized elliptic operators, $F$; then the continuation operator $C_{\left(\alpha, \alpha_{0}\right)}$ becomes elliptic as well.

\section{Construction of continuation}

In seismic applications $Y$ and $X$ are often different spaces, with different physical roles. As before, we will refer to $F$ as demigration (modelling) and to $F^{*}$ as migration (imaging) operators. (In the presence of caustics, one requires an extension [32] to ensure that $F$ belongs to $\mathcal{C}$. Originally, the dimension of $Y$ exceeds the dimension of $X$, while it is assumed that the projection of the canonical relation of $F$ into $T^{*} Y \backslash 0$ is an injection.) In general, the amplitude and phase of $F$ are determined by a model $m \in M$, where $M$ stands for a model (such as background velocity) or configuration (such as source-receiver acquisition) space. A curve, $m[\alpha]$, in $M$ thus defines a one-parameter family of demigration FIOs $F(\alpha) \in \mathcal{C}$ with amplitudes $a=a(\alpha ; y, \xi)$ and phase functions $\phi=\phi(\alpha ; y, x, \xi)$, cf. (12)-(13). We denote the canonical relation of $F(\alpha)$ by $\Lambda_{\alpha}$ and the one of $F(\alpha)^{*}$ by $\Lambda_{\alpha}^{*}$.

Continuation operators $C_{\left(\alpha, \alpha_{0}\right)}$ defined in (22) belong to $\mathcal{C}$ with $Y=X$. Examples are image continuation following a path of background media (of which all but one are 'incorrect'), and data continuation with source-receiver offset. Suppose that (i) given data $u(y)$, an image, $w(x)=w\left(\alpha_{0}, x\right)$, has been obtained in a model parameterized by $\alpha_{0}$, or (ii) given an image $w(x)$, data $u(y)=u\left(\alpha_{0}, y\right)$ have been obtained in a model parameterized by $\alpha_{0}$, or (iii) data $u(y)=u\left(\alpha_{0}, y\right)$ have been acquired in an acquisition geometry parameterized by $\alpha_{0}$. Suppose that models or acquisition geometries of interest can be connected along a path parameterized by $\alpha$ taking values in an interval, $I=\left[\alpha_{1}, \alpha_{2}\right] \subset \mathbb{R}$, containing $\alpha_{0}$. The framework developed in the previous section can be applied to directly 'continuing' $w\left(\alpha_{0}, x\right)$ or $u\left(\alpha_{0}, y\right)$ along such 
a path; we arrive at continuation operators, $C^{Y}, C^{X}$, such that

$$
u(\alpha, y)=\left(C_{\left(\alpha, \alpha_{0}\right)}^{Y} u\left(\alpha_{0}, .\right)\right)(y), \quad w(\alpha, x)=\left(C_{\left(\alpha, \alpha_{0}\right)}^{X} w\left(\alpha_{0}, .\right)\right)(x),
$$

with $\alpha_{1} \leq \alpha_{0} \leq \alpha \leq \alpha_{2}$. Then composition (22) can be specified for these two cases:

$$
C_{\left(\alpha, \alpha_{0}\right)}^{Y}=F(\alpha) F\left(\alpha_{0}\right)^{*}, \quad C_{\left(\alpha, \alpha_{0}\right)}^{X}=F(\alpha)^{*} F\left(\alpha_{0}\right) .
$$

In the remainder of the paper, we focus on $C_{\left(\alpha, \alpha_{0}\right)}^{X}$.

In the previous section we have formulated (i) sufficient and necessary conditions to be able to develop a continuation theory, (ii) proved the existence of hyperbolic evolution equations that describe the process of continuation. This formulation naturally brings us to associated notions of global Hamiltonians and the existence of continuation bicharacteristics (cf. (23)-(25)).

A specific task in seismic applications is the construction of evolution equations of the type (20) for given $F(\alpha)$. We note that in special cases corresponding with simple media the pseudodifferential equation can be written in partial differential form (see Goldin [23] for such constructions using contact geometry). To obtain $P(\alpha)$ may require numerical computations. However, the computational gain in continuation is achieved by avoiding the re-application of $F^{*}$ to all the data.

We discuss three methods of construction. The first approach (method 1) comprises the straightforward composition of 'migration' and 'demigration' operators through their canonical relations. In the second approach (method 2) we derive the evolution operator in (20) from the phase function of 'migration' operator $F(\alpha)^{*}$. In the third approach (method 3), we obtain the (principal part of the) evolution operator in (20) from the combined phase function of the composition $F(\alpha)^{*} F(\alpha)$ (which may be viewed as a generalization of considerations in [14]).

\subsection{Method 1. Continuation by composition}

The canonical relation for the continuation operator $C_{\left(\alpha, \alpha_{0}\right)}^{X}$ (cf. (28)) follows the composition (cf. (10)) as the graph condition is satisfied for both $F(\alpha)$ and $F(\alpha)^{*}$ :

$$
\begin{aligned}
& \Lambda_{\left(\alpha, \alpha_{0}\right)}^{C^{X}}=\Lambda_{\alpha}^{*} \circ \Lambda_{\alpha_{0}}=\left\{\left(x, \xi ; x_{0}, \xi_{0}\right) \mid(x, \xi ; y, \eta) \in \Lambda_{\alpha}^{*}\right. \\
& \left.\quad \text { and }\left(y, \eta ; x_{0}, \xi_{0}\right) \in \Lambda_{\alpha_{0}} \text { for some }(y, \eta) \in T^{*} Y \backslash 0\right\} .
\end{aligned}
$$

Note that the amplitude can be obtained following the corresponding composition calculus. Also note that canonical relations $\Lambda_{\left(\alpha, \alpha_{0}\right)}^{C^{X}}, \Lambda_{\alpha}^{*}$ and $\Lambda_{\alpha_{0}}$ are associated with canonical transformations $\Sigma_{\left(\alpha, \alpha_{0}\right)}^{C}, \Sigma_{\alpha}^{F^{*}}$ and $\Sigma_{\alpha_{0}}^{F}$ correspondingly (cf. 11). The curves defined by the canonical 
transformation determine a vector field on $T\left(T^{*} X \backslash 0\right)$ :

$$
V_{\alpha}(x, \xi)=\frac{\mathrm{d}}{\mathrm{d} \alpha} \Sigma_{\left(\alpha, \alpha_{0}\right)}^{C}\left(x_{0}, \xi_{0}\right)=\frac{\mathrm{d}}{\mathrm{d} \alpha} \Sigma_{\alpha}^{F^{*}}\left(y_{0}, \eta_{0}\right),
$$

where $(x, \xi)=\Sigma_{\left(\alpha, \alpha_{0}\right)}^{C}\left(x_{0}, \xi_{0}\right),\left(y_{0}, \eta_{0}\right)=\Sigma_{\alpha_{0}}^{F}\left(x_{0}, \xi_{0}\right)$ and we used that the canonical transformation $\Sigma_{\alpha_{0}}^{F}$ (corresponding to $\Lambda_{\alpha_{0}}$ ) does not depend on $\alpha$. (Right equality in (30) manifests the fact that vector field for continuation operator can be obtained applying perturbation argument to a 'migration' operator $F(\alpha)^{*}$ (thus getting $\frac{\mathrm{d}}{\mathrm{d} \alpha} \Sigma_{\alpha}^{F^{*}}$ ).) Here $V_{\alpha}:=\left(V_{1}, V_{2}\right)$ is the tangent vector to the curve $\left(x\left(\alpha, x_{0}, \xi_{0}\right), \xi\left(\alpha, x_{0}, \xi_{0}\right)\right)=\Sigma_{\left(\alpha, \alpha_{0}\right)}^{C}\left(x_{0}, \xi_{0}\right)$ in $T^{*} X$,

$$
\frac{\mathrm{d} x}{\mathrm{~d} \alpha}=V_{1}(\alpha, x, \xi), \quad \frac{\mathrm{d} \xi}{\mathrm{d} \alpha}=V_{2}(\alpha, x, \xi) .
$$

If $\omega$ is the canonical symplectic form on $T^{*} X \backslash 0$, then, by definition,

$$
\Sigma_{\left(\alpha, \alpha_{0}\right)}^{*} \omega=\omega
$$

where $\Sigma_{\left(\alpha, \alpha_{0}\right)}^{*}$ stands for a pull-back of $\omega$ by transformation $\Sigma_{\left(\alpha, \alpha_{0}\right)}^{C}$. Differentiating (32), we get [42]

$$
0=\frac{\mathrm{d}}{\mathrm{d} \alpha} \Sigma_{\left(\alpha, \alpha_{0}\right)}^{*} \omega(\cdot)=\Sigma_{\left(\alpha, \alpha_{0}\right)}^{*}\left(\mathrm{~d} \omega\left(\cdot, V_{\alpha}\right)\right)
$$

But then

$$
\mathrm{d} \omega\left(\cdot, V_{\alpha}\right)=0
$$

Then there exists a $C^{\infty}$ function $q=q(\alpha, x, \xi)$ such that $\omega\left(\cdot, V_{\alpha}\right)=\mathrm{d}_{(x, \xi)} q$, and $q$ can be computed by the formula

$$
q(\alpha, x, \xi)=\sum_{j=1}^{n_{X}} \int_{0}^{1}\left[V_{1 j}(\alpha, t x, t \xi) \xi_{j}-V_{2 j}(\alpha, t x, t \xi) x_{j}\right] \mathrm{d} t
$$

see [43, Theorem 4.18]. We have assumed that the manifold $T^{*} X$ is essentially an open set in $\mathbb{R}^{2 n_{X}}$, so that we can apply Poincaré's lemma (whence the form $\omega(\cdot, V)$ is exact). It is clear that if $V_{\alpha}$ is smooth in $\alpha$, so is $q$. It follows that $q$ coincides with $p_{1}$ up to an additive constant. It means that given vector field for the continuation operator one can find the continuation Hamiltonian $p_{1}$.

Continuation Hamiltonian from integrable vector field. In various applications (see Section 5.3), a construction (based on perturbation arguments) leads directly to equations of the type (31), that is, a vector field $V_{\alpha}$. Then one may question the applicability of the theory presented here, in particular, the existence of a global Hamiltonian (cf. (23)). To guarantee the validity of (33), one checks whether the Lie derivative, $\mathcal{L}_{V_{\alpha}} \omega=0$. 
In local coordinates, $(x, \eta)$, and $V_{\alpha}=\left(V_{1}(\alpha, x, \xi), V_{2}(\alpha, x, \xi)\right)$, with

$$
V_{1}(\alpha, x, \xi)=\left(V_{11}(\alpha, x, \xi), \ldots, V_{1 n}(\alpha, x, \xi)\right) \text { and } V_{2}(\alpha, x, \xi)=\left(V_{21}(\alpha, x, \xi), \ldots, V_{2 n}(\alpha, x, \xi)\right)
$$

being the $\partial_{x}$ and $\partial_{\xi}$ components of $V_{\alpha}$ respectively, equation (33) is equivalent to

$$
\begin{gathered}
\frac{\partial V_{2 j}}{\partial x_{k}}=\frac{\partial V_{2 k}}{\partial x_{j}}, j \neq k, \quad \frac{\partial V_{1 j}}{\partial \xi_{k}}=\frac{\partial V_{1 k}}{\partial \xi_{j}}, j \neq k, \\
\frac{\partial V_{2 j}}{\partial x_{k}}=-\frac{\partial V_{1 k}}{\partial \xi_{j}}, \quad \text { for all } j, k .
\end{gathered}
$$

If $\mathrm{d} \omega\left(\cdot, V_{\alpha}\right)=0$, that is, if equations (35) hold, then there is a global Hamiltonian (34) generating this vector field.

\subsection{Method 2. Evolution operator from 'migration' operator}

The derivative $\partial_{\alpha} C_{\left(\alpha, \alpha_{0}\right)}^{X}$ is determined by $\partial_{\alpha} F(\alpha)^{*}$ : It is immediate that (cf. (21))

$$
\partial_{\alpha} C_{\left(\alpha, \alpha_{0}\right)}^{X}=\mathrm{i} P(\alpha) C_{\left(\alpha, \alpha_{0}\right)}^{X}=\mathrm{i} P(\alpha) F(\alpha)^{*} F\left(\alpha_{0}\right) ;
$$

because $F\left(\alpha_{0}\right)$ is invertible it follows that

$$
\partial_{\alpha} F(\alpha)^{*}=\mathrm{i} P(\alpha) F(\alpha)^{*} .
$$

Thus the perturbation of the image continuation operator $C_{\left(\alpha, \alpha_{0}\right)}^{X}$ is completely determined by the perturbation of migration operator $F(\alpha)^{*}$.

Since the canonical relation of $F(\alpha)^{*}$ is a graph, it admits coordinates $(x, \eta)$ and a generating function $\widetilde{S}=\widetilde{S}(\alpha ; x, \eta)$ (cf. (12)-(13)). The kernel of $F(\alpha)^{*}$ then admits the representation

$$
A_{F(\alpha)^{*}}(x, y)=\int \widetilde{a}(\alpha ; x, \eta) \exp [\mathrm{i} \widetilde{\phi}(\alpha ; x, y, \eta)] \mathrm{d} \eta, \quad \widetilde{\phi}(\alpha ; x, y, \eta)=\widetilde{S}(\alpha ; x, \eta)-\langle\eta, y\rangle .
$$

To leading order, the kernel of $\partial_{\alpha} F(\alpha)^{*}$ has the representation (cf. (19))

$$
\text { i } \int \partial_{\alpha} \widetilde{S}(\alpha ; x, \eta) \widetilde{a}(\alpha ; x, \eta) \exp [\mathrm{i} \widetilde{\phi}(\alpha ; x, y, \eta)] \mathrm{d} \eta \text {. }
$$

We introduce the change of coordinates, $(x, \eta) \rightarrow(x, \xi)$, by solving the equation

$$
\xi=\partial_{x} \widetilde{S}(\alpha ; x, \eta)
$$

for $\eta=\eta(\alpha ; x, \xi)$. The principal symbol of pseudodifferential $\widetilde{P}(\alpha)$ then follows to be

$$
\widetilde{p}_{1}(\alpha, x, \xi)=\partial_{\alpha} \widetilde{S}(\alpha ; x, \eta(\alpha ; x, \xi)) .
$$


Indeed, applying the composition rule for a pseudodifferential operator with an FIO [25, Ch. VIII, p.465],

$$
\widetilde{P}\left(\alpha, x, D_{x}\right) A_{F(\alpha)^{*}}(x, y)=\int \widetilde{p}\left(\alpha, x, \partial_{x} \widetilde{S}(\alpha ; x, \eta)\right) \widetilde{a}(\alpha ; x, \eta) \exp [\mathrm{i} \widetilde{\phi}(\alpha ; x, y, \eta)] \mathrm{d} \eta,
$$

and using $\partial_{\alpha} \widetilde{S}\left(\alpha ; x, \eta\left(\alpha ; x, \partial_{x} \widetilde{S}(\alpha ; x, \eta)\right)\right)=\partial_{\alpha} \widetilde{S}(\alpha ; x, \eta)$, we recover (39) and thus get (41). Since $\widetilde{S}$ is homogeneous of degree 1 in $\eta, \widetilde{p}_{1}$ is a symbol of order 1 .

The continuation bicharacteristics $x(\alpha), \xi(\alpha)$ are the solution to Hamilton system (25), which, with (41), attains the form

$$
\begin{aligned}
& \frac{\mathrm{d} x}{\mathrm{~d} \alpha}=-\left[\partial_{\xi} \eta(\alpha ; x, \xi)\right] \cdot\left(\partial_{\eta} \partial_{\alpha} \widetilde{S}\right)(\alpha ; x, \eta(\alpha ; x, \xi)) \\
& \frac{\mathrm{d} \xi}{\mathrm{d} \alpha}=\partial_{x} \partial_{\alpha} \widetilde{S}(\alpha ; x, \eta(\alpha ; x, \xi))+\left[\partial_{x} \eta(\alpha ; x, \xi)\right] \cdot\left(\partial_{\eta} \partial_{\alpha} \widetilde{S}\right)(\alpha ; x, \eta(\alpha ; x, \xi))
\end{aligned}
$$

\subsection{Method 3. Evolution operator from 'migration'-'demigration' composition}

We use the oscillatory integral representation for the kernel of $F(\alpha)$ obtained from the one for the kernel of $F(\alpha)^{*}$. In terms of phase functions, the composition of canonical relations (cf. (10)) in $\Lambda_{\left(\alpha, \alpha_{0}\right)}^{C^{X}}$ follows the construction of the stationary point set (cf. (7)-(8)) for $\widetilde{\phi}(\alpha ; x, y, \eta)-$ $\widetilde{\phi}\left(\alpha_{0} ; x_{0}, y, \eta^{\prime}\right)$ :

$$
\begin{aligned}
\partial_{\eta} \widetilde{\phi}(\alpha ; x, y, \eta)=0, & \partial_{\eta^{\prime}} \widetilde{\phi}\left(\alpha_{0} ; x_{0}, y, \eta^{\prime}\right)=0, \\
& \partial_{y}\left[\widetilde{\phi}(\alpha ; x, y, \eta)-\widetilde{\phi}\left(\alpha_{0} ; x_{0}, y, \eta^{\prime}\right)\right]=0,
\end{aligned}
$$

on which $\left(x, \partial_{x} \widetilde{\phi}(\alpha ; x, y, \eta) ; x_{0}, \partial_{x_{0}} \widetilde{\phi}\left(\alpha_{0} ; x_{0}, y, \eta^{\prime}\right)\right)$ determines the points in $\Lambda_{\alpha}^{*} \circ \Lambda_{\alpha_{0}}=\Lambda_{\left(\alpha, \alpha_{0}\right)}^{C^{X}}$. We eliminate the bottom equation, and substitute its solution, $\eta^{\prime}=\eta$, in the top equations, that is, $\partial_{\eta}[\widetilde{S}(\alpha ; x, \eta)]=y=\partial_{\eta}\left[\widetilde{S}\left(\alpha ; x_{0}, \eta\right)\right]$, whence

$$
\partial_{\eta}\left[\widetilde{S}(\alpha ; x, \eta)-\widetilde{S}\left(\alpha_{0} ; x_{0}, \eta\right)\right]=0 .
$$

With $x(\alpha)$ denoting a continuation characteristic as before, while perturbing $\alpha$ about $\alpha_{0}$ and $x=x(\alpha)$ about $x_{0}=x\left(\alpha_{0}\right)$, it follows that

$$
\partial_{\alpha} \partial_{\eta} \widetilde{S}(\alpha ; x, \eta)+\frac{\mathrm{d} x}{\mathrm{~d} \alpha} \cdot\left[\partial_{x} \partial_{\eta} \widetilde{S}(\alpha ; x, \eta)\right]=0, \quad x=x(\alpha) .
$$

Because $\partial_{x} \partial_{\eta} \widetilde{S}$ is non-singular, this is a system of $n_{X}$ equations that provides a solution for $\frac{\mathrm{d} x}{\mathrm{~d} \alpha}$ for each $\eta\left(=-\partial_{y} \widetilde{\phi}\right)$. With initial condition, $x\left(\alpha_{0}, y_{0}, \eta_{0}\right)=x_{0}$, it holds true that $\widetilde{S}\left(\alpha_{0} ; x_{0}, \eta_{0}\right)-$ $\left\langle\eta_{0}, y_{0}\right\rangle=0$. 
It is straightforward to verify that the solution to (47) coincides with (43):

$$
\frac{\mathrm{d} x}{\mathrm{~d} \alpha}=-\left[\partial_{\eta} \partial_{x} \widetilde{S}\right]^{-1} \cdot\left(\partial_{\alpha} \partial_{\eta} \widetilde{S}\right)=-\left[\partial_{\eta} \xi\right]^{-1} \cdot\left(\partial_{\eta} \partial_{\alpha} \widetilde{S}\right), \quad \text { using that } \xi=\partial_{x} \widetilde{S}
$$

However, this construction does not directly provide the underlying Hamiltonian, and a full set of Hamilton equations (43)-(44). For deriving Hamiltonian one needs to use techniques described in previous sections (methods 1 and 2).

\section{Examples}

Here, we connect some known procedures for continuation to the general framework developed in this paper. In particular, we discuss the velocity continuation of images and isochrons in common-offset Kirchhoff migration [14,12], and the continuation of offset image gathers $[18,19]$ in the absence of caustics. We derive explicit formulae for the Hamiltonians in the constant velocity case. A family of migration-demigration operators is defined by a smooth family of background velocities $v[\alpha]$. In the case of constant velocity, $v[\alpha] \equiv v=$ const and $v$ itself plays the role of $\alpha$.

\subsection{Common-offset image continuation characteristics assuming the absence of caustics}

In this subsection we apply methods 2 and 3 (Sections 4.2 and 4.3) to common-offset migration of seismic data. In Kirchhoff migration, operator $F(\alpha)^{*}$ transforms data $u(y)$ into an image $w(x)$. then We assume the absence of caustics. On the acquisition manifold we introduce the following coordinates: $y=\left(t, y^{\prime}\right)$, where $t$ is the time, $y^{\prime}=(r+s) / 2$ is the source-receiver midpoint, and $h=(r-s) / 2$ is half-offset; $s$ indicates a source position and $r$ indicates a receiver position, see Fig. 1. In common offset ${ }^{4}$ migration we consider $h$ to be a set of parameters so that $F(\alpha)^{*}: u\left(t, y^{\prime}\right) \rightarrow w(x)$.

A phase function $\widetilde{\phi}$ for the oscillatory integral representation of the kernel of operator $F(\alpha)^{*}$ can be chosen of the form

$$
\widetilde{\phi}\left(\alpha ; x, t, y^{\prime}, \tau\right)=-\phi\left(\alpha ; t, y^{\prime}, x, \tau\right), \quad \phi\left(\alpha ; t, y^{\prime}, x, \tau\right)=\tau\left(T\left(\alpha ; y^{\prime}, x\right)-t\right)
$$

in which $T\left(\alpha ; y^{\prime}, x\right)$ denotes the 'two-way' traveltime along a broken ray connecting a receiver

${ }^{4}$ In three-dimensional configurations, offset is a two-dimensional vector; representing this vector in polar coordinates, one refers to the angular coordinate as azimuth. 
at $r$ to a source at $s$ (defining $y^{\prime}$ ) via the scattering point $x$, that is,

$$
T\left(\alpha ; y^{\prime}, x\right)=t_{s}\left(\alpha ; y^{\prime}-h, x\right)+t_{r}\left(\alpha ; y^{\prime}+h, x\right),
$$

if $t_{s}$ denotes the traveltime along a source ray (connecting $s=y^{\prime}-h$ with $x$ ) and $t_{r}$ denotes the traveltime along a receiver ray (connecting $r=y^{\prime}+h$ with $x$ ), see Fig. 1 . In the above, $\phi$ is the phase function for demigration operator $F(\alpha) ; \tau$ is the only phase variable (cf. (9)). The propagation of singularities by $F(\alpha)^{*} F\left(\alpha_{0}\right)$ is illustrated in Fig. 1.

First we apply method 3 (Section 4.3) with phase functions given in (49). This leads to equations for velocity continuation characteristics, repeating the reasoning in (45)-(47) (with only one phase variable, $\tau$ )

$$
\partial_{\left(\tau, y^{\prime}\right)}\left(\tau \partial_{\alpha} T\left(\alpha ; y^{\prime}, x\right)\right)+\frac{\mathrm{d} x}{\mathrm{~d} \alpha} \cdot\left[\partial_{x} \partial_{\left(\tau, y^{\prime}\right)}\left(\tau T\left(\alpha ; y^{\prime}, x\right)\right)\right]=0,
$$

see $[14,(\mathrm{C}-4)]$. The quantities $\left[\partial_{x} \partial_{\left(\tau, y^{\prime}\right)}(\tau T)\right]$ and $\partial_{\left(\tau, y^{\prime}\right)} \partial_{\alpha}(\tau T)$ can be obtained by methods of dynamic ray tracing and ray perturbation. The matrix, $\left[\partial_{\left(\tau, y^{\prime}\right)} \partial_{x}(\tau T)\right]$, using that $\xi=\partial_{x}(\tau T)$, has a determinant which appears in 'true-amplitude' common-offset imaging based on the generalized Radon transform and has been attributed to Beylkin [44, p.223].

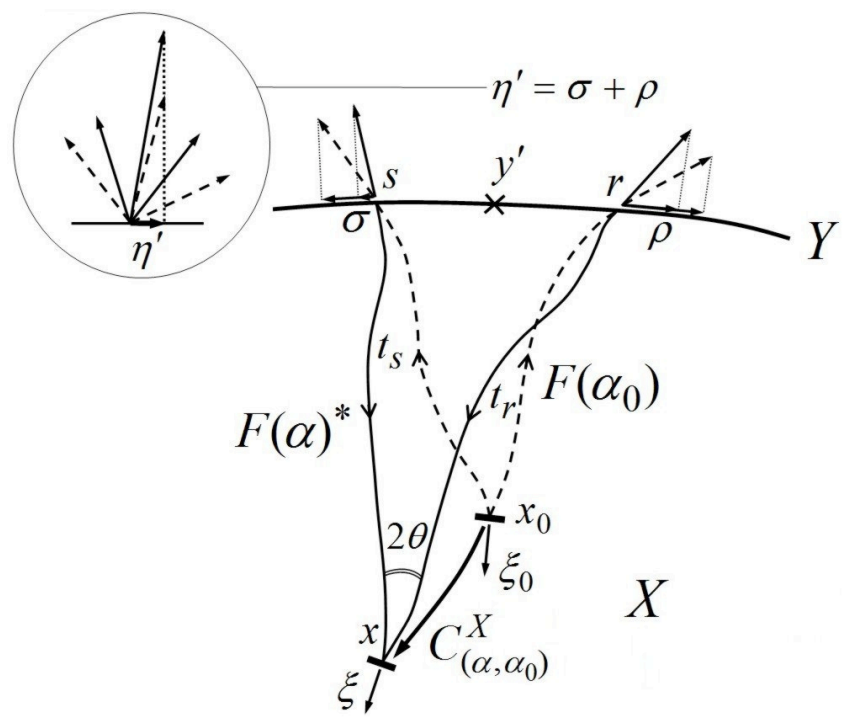

Fig. 1. The continuation $C_{\left(\alpha, \alpha_{0}\right)}^{X}=F(\alpha)^{*} F\left(\alpha_{0}\right)$ in the case of common-offset migration. Pairs of rays connecting local reflector points and dips, $\left(x_{0}, \xi_{0}\right)$ and $(x, \xi)$, with acquisition midpoint, traveltime and slope, $\left(y^{\prime}, t, \eta^{\prime}\right)$, illustrate demigration (dashed lines) and migration (solid lines) with different background velocities.

To obtain the continuation Hamiltonian we invoke method 2 from Section 4.2. We apply Appendix A to the generating function $S^{\prime}\left(\alpha ; x, y^{\prime}, \tau\right)=\tau T\left(\alpha ; y^{\prime}, x\right)$, to obtain $\widetilde{S}(\alpha ; x, \eta)=$ $\left.\left(\tau T\left(\alpha ; y^{\prime}, x\right)+\left\langle\eta^{\prime}, y^{\prime}\right\rangle\right)\right|_{y^{\prime}=y^{\prime}(\alpha ; x, \eta)}$ (cf. (38)) with $\eta=\left(\tau^{\prime}, \eta^{\prime}\right)$; here, the stationary points 
$y^{\prime}(\alpha ; x, \eta)$ are found from the equations

$$
\tau \partial_{y^{\prime}} T\left(\alpha ; y^{\prime}, x\right)=-\eta
$$

After substituting the solution of (52) into (40) we find that $\eta^{\prime}=\eta^{\prime}(\alpha ; x, \xi)$ and $\tau=\tau(\alpha ; x, \xi)$ solve

$$
\begin{aligned}
\xi & =\partial_{x} \widetilde{S}(\alpha ; x, \eta) \\
& =\tau \partial_{x} T\left(\alpha ; y^{\prime}(\alpha ; x, \eta), x\right)+\tau \partial_{x} y^{\prime}(\alpha ; x, \eta) \cdot \partial_{y^{\prime}} T\left(\alpha ; y^{\prime}(\alpha ; x, \eta), x\right)+\partial_{x} y^{\prime}(\alpha ; x, \eta) \cdot \eta^{\prime} \\
& =\tau \partial_{x} T\left(\alpha ; y^{\prime}(\alpha ; x, \eta), x\right) .
\end{aligned}
$$

One can combine equations (52) and (53) and solve directly

$$
\tau \partial_{x} T\left(\alpha ; y^{\prime}, x\right)=\xi
$$

for $y^{\prime}(\alpha ; x, \xi)$ and $\tau(\alpha ; x, \xi)$ (a similar phase variable transformation appeared in [45] while developing map migration using curvelets).

The Hamiltonian for continuation bicharacteristics, using (41), then follows to be

$$
\mathcal{H}\left(\alpha, x, \xi_{\alpha}, \xi\right)=\xi_{\alpha}-\underbrace{\tau(\alpha ; x, \xi) \partial_{\alpha} T\left(\alpha ; y^{\prime}(\alpha ; x, \xi), x\right)}_{=\widetilde{p}_{1}(\alpha, x, \xi)} .
$$

We will evaluate this Hamiltonian in the case of constant background velocities in the next subsection. We note that along continuation bicharacteristics, $\xi_{\alpha}=\tau(\alpha ; x, \xi) \partial_{\alpha} T\left(\alpha ; y^{\prime}(\alpha ; x, \xi), x\right)$.

\subsection{Global Hamiltonian for constant velocity continuation}

Here, we apply method 2 (Section 4.2) to construct the global Hamiltonian for velocity continuation. We let the points $x$ now have coordinates $(x, z)$, while all $s, r, y^{\prime}$ and $h$ lie in the horizontal plane, $z=0 ; v$ is identified with $\alpha$. We get (cf. (50))

$$
\begin{aligned}
& t_{s}\left(v ; y^{\prime}-h, x, z\right)=\frac{\rho_{s}\left(y^{\prime}, x, z\right)}{v}, t_{r}\left(v ; y^{\prime}+h, x, z\right)=\frac{\rho_{r}\left(y^{\prime}, x, z\right)}{v}, \\
& \rho_{s}\left(y^{\prime}, x, z\right)=\sqrt{\left(x-y^{\prime}+h\right)^{2}+z^{2}}, \quad \rho_{r}\left(y^{\prime}, x, z\right)=\sqrt{\left(x-y^{\prime}-h\right)^{2}+z^{2}} .
\end{aligned}
$$

The coordinates, and three, evolving, isochrons (half ellipses, in this case) are shown in Fig. 4. By differentiating (50) with (56) with respect to $v$, we obtain (63) for the constant background media case:

$$
-\frac{\rho_{s}+\rho_{r}}{v^{2}}+\left(\frac{\partial_{x} \rho_{s}+\partial_{x} \rho_{r}}{v}\right) \frac{\mathrm{d} x}{\mathrm{~d} v}+\left(\frac{\partial_{z} \rho_{s}+\partial_{z} \rho_{r}}{v}\right) \frac{\mathrm{d} z}{\mathrm{~d} v}=0,
$$


where simply

$$
\partial_{x} \rho_{s}=\frac{x-y^{\prime}+h}{\rho_{s}}, \quad \partial_{z} \rho_{s}=\frac{z}{\rho_{s}}
$$

and similarly for $\partial_{x} \rho_{r}$ and $\partial_{z} \rho_{r}$.

Then equations (54) take the following form:

$$
\frac{\left(x-y^{\prime}-h\right)}{v \rho_{r}}+\frac{\left(x-y^{\prime}+h\right)}{v \rho_{s}}=\frac{\xi}{\tau}, \frac{z}{v \rho_{r}}+\frac{z}{v \rho_{s}}=\frac{\zeta}{\tau}
$$

were $(\xi, \zeta)$ are variables dual to $(x, z)$. Eliminating $\tau$ results in

$$
y^{\prime}(x, z, \xi, \zeta)=x+\frac{z\left(\zeta^{2}-\xi^{2}\right)-\sqrt{(2 h \xi \zeta)^{2}+z^{2}\left(\xi^{2}+\zeta^{2}\right)^{2}}}{2 \xi \zeta},
$$

Following (41), (55), we have (with the second equation in (59)),

$$
\begin{array}{r}
\widetilde{p}(v, x, z, \xi, \zeta)=\tau \partial_{v} T=-\left.\frac{\tau}{v^{2}}\left(\rho_{r}+\rho_{s}\right)\right|_{y^{\prime}=y^{\prime}(x, z, \xi, \zeta)}=-\left.\frac{\zeta}{z v} \rho_{r} \rho_{s}\right|_{y^{\prime}=y^{\prime}(x, z, \xi, \zeta)} \\
=-\frac{\xi^{2}+\zeta^{2}}{2 v \xi^{2} \zeta} \sqrt{(2 h \xi \zeta)^{2}+2 z^{2}\left(\xi^{4}+\zeta^{4}\right)+2 z\left(\xi^{2}-\zeta^{2}\right) \sqrt{(2 h \xi \zeta)^{2}+z^{2}\left(\xi^{2}+\zeta^{2}\right)^{2}}}
\end{array}
$$

yielding the symbol of the evolution operator. We can further simplify (61) and obtain the global Hamiltonian for velocity continuation (cf. (55); an alternative form of this Hamiltonian was derived by Fomel [46])

$$
\mathcal{H}\left(v, x, z, \xi_{v}, \xi, \zeta\right)=\xi_{v}+\frac{\xi^{2}+\zeta^{2}}{2 v \xi^{2} \zeta}\left[z\left(\xi^{2}-\zeta^{2}\right)+\sqrt{(2 h \xi \zeta)^{2}+z^{2}\left(\xi^{2}+\zeta^{2}\right)^{2}}\right] .
$$

Remark. On the slowness surface associated to the Hamiltonian in (62), we have that if $\xi_{v}>0$ then $\zeta<0$ (we note that the expression in square brackets in (62) is always non-negative, so that the sign of the second term is controlled by the sign of $\zeta$ ), and, hence, if $\xi_{v}<0$ then $\zeta>0$. The slowness surface ( $\mathcal{H}=0$ for given $(x, z)$ ) is depicted in Fig. 2 a) (where we introduce the normalized vector components, $k_{x}=\xi / \xi_{v}$ and $k_{z}=\zeta / \xi_{v}$ ), while the group velocities (cf. (25) or (43)) are shown in Fig. 2 b). We consider two cases: Small offset $(h / z=0.5)$ and large offset $(h / z=1.7)$, while setting $z=1$ and $v=1$ in the computation. For small offsets, the slowness surface approaches a circle and the group velocity surface approaches a parabola. For large offsets, the slowness surface develops inflection points, leading to cusps in the group velocity surface. Note that the group velocity surface corresponds to an 'instantaneous front' generated at a point in the initial image; see [6,11]. 


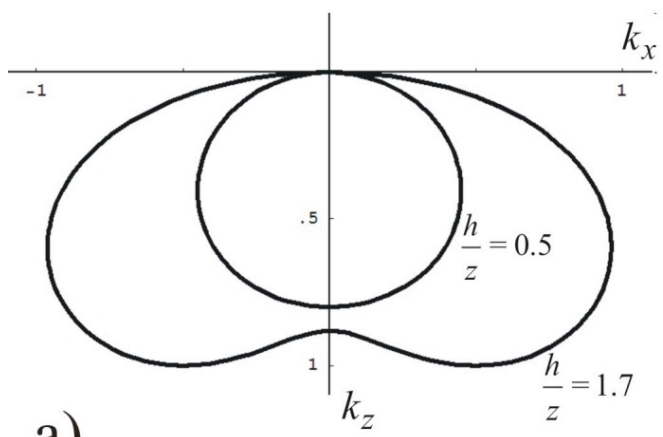

a)

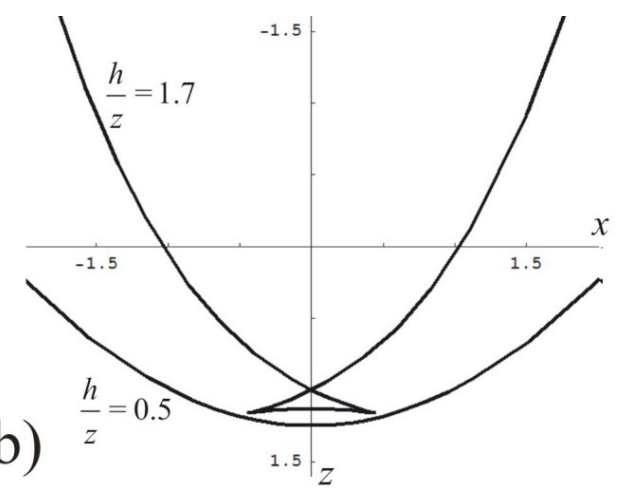

Fig. 2. (a) - Slowness surface for small offset $(h / z=0.5)$ and large offset $(h / z=1.7)$; (b) - group velocity surface for small offset $(h / z=0.5)$ and large offset $(h / z=1.7)$.

Making use of the global Hamiltonian (62), we illustrate common-offset image continuation and the notion of continuation characteristics. In Fig. 3 (left) we show continuation characteristics calculated for a segment (in bold) of a planar (line) reflector. The initial (correct) commonoffset image corresponds to a background velocity $v=1 \mathrm{~km} / \mathrm{s}$; common-offset migrations for different values of $h$ will produce the same image. Continuation characteristics (thin lines) take off from the original image and terminate at an image for $v=1.3 \mathrm{~km} / \mathrm{s}$ (straight line segment to the left) and an image for $v=0.5 \mathrm{~km} / \mathrm{s}$ (straight line segment to the right). Thin solid lines represent continuation characteristics corresponding to offset $h=0.1 \mathrm{~km}$, and dashed thin lines represent continuation characteristics corresponding to $h=0.7 \mathrm{~km}$. In Fig. 3 (right) the reflector, and the initial (correct) common-offset image, are parabolic. The correct (and initial) background velocity is $v=1 \mathrm{~km} / \mathrm{s}$; the image is continued to $v=1.06 \mathrm{~km} / \mathrm{s}$. Even in this simple model, we observe the formation of caustics.
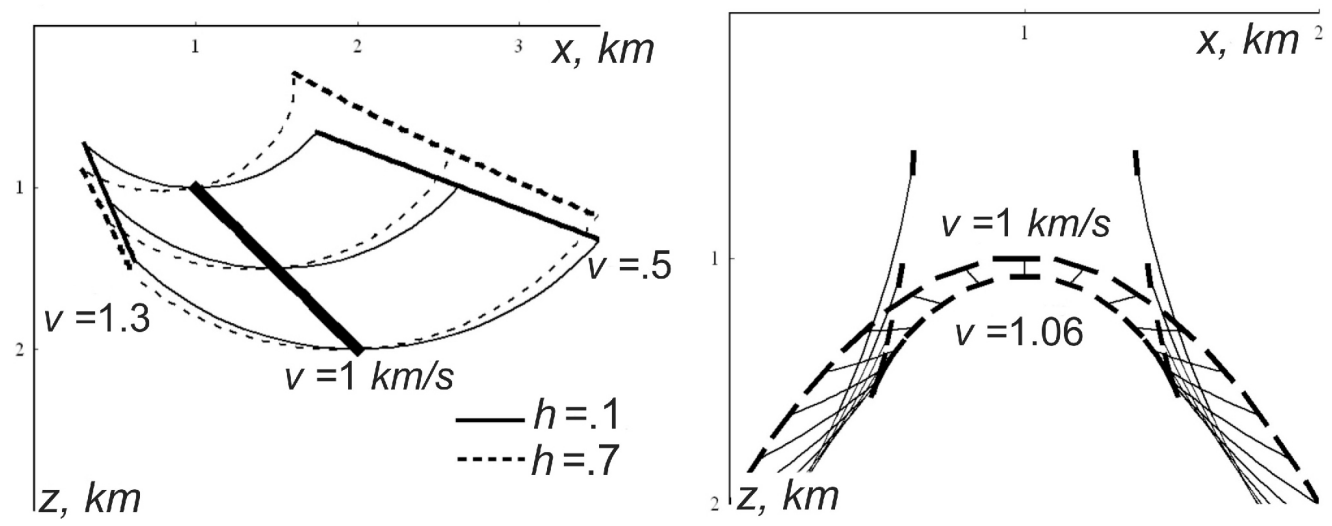

Fig. 3. Common-offset image continuation and continuation characteristics. Left: plane reflector (solid line segment); thin solid lines indicate continuation characteristics for $h=0.1 \mathrm{~km}$, while thin dashed lines indicate continuation characteristics for $h=0.7 \mathrm{~km}$. Right: parabolic reflector; thin solid lines indicate continuation characteristics. 


\section{3 'Velocity rays' as curves connecting evolving isochrons}

Isochrons, generated by $F(\alpha)^{*}$, are given by

$$
\mathcal{W}\left(\alpha ; t, y^{\prime}\right)=\left\{(x, z) \in X \mid T\left(\alpha ; y^{\prime}, x, z\right)=t\right\}
$$

see Section 2. 'Velocity rays' were introduced in the literature as curves connecting isochrons evolving with $\alpha$ (cf. [3]). Such curves are written as $(x(\alpha), z(\alpha))$, and must then satisfy

$$
\partial_{\alpha} T=-\partial_{(x, z)} T \cdot \frac{\mathrm{d}(x, z)}{\mathrm{d} \alpha}
$$

(keeping $\left(t, y^{\prime}\right)$ fixed). Since, for now, we have only one equation, we will have to supplement it with another equation for $\frac{\mathrm{d} x}{\mathrm{~d} \alpha}$ and $\frac{\mathrm{d} z}{\mathrm{~d} \alpha}$ to be determined. In this subsection, we discuss four different supplementary equations from the literature, each leading to a notion of velocity 'rays', that is, curves $(x(\alpha), z(\alpha))$. These supplementary equations were not obtained by methods outlined here, whence we need to check the integrability condition (cf. (35)) to verify whether the implied curves can be associated with the continuation operator.

1. Liu and Bleistein [18]: vertical 'ray'. The authors assume that the curves that connect an initial with a perturbed isochron are vertical: $\frac{\mathrm{d} x}{\mathrm{~d} \alpha}=0$; in constant background media,

$$
\frac{\mathrm{d} x}{\mathrm{~d} v}=0
$$

Solving equations (57) and (64), one obtains $\frac{\mathrm{d} z}{\mathrm{~d} v}=\frac{\rho_{s} \rho_{r}}{v z}$ (as in $[18,(13)]$ ) describing vertical 'rays'. Corresponding curves are illustrated, and indexed by 1, in Fig. 4.

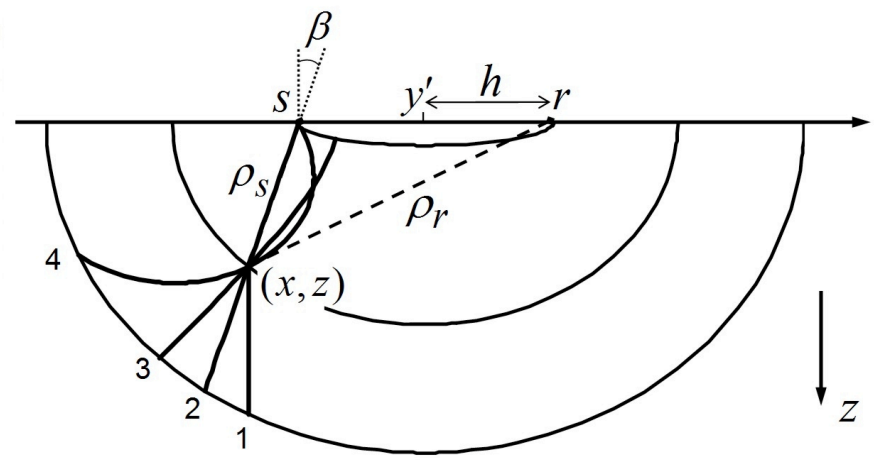

Fig. 4. Three isochrons for fixed $\left(t, y^{\prime}, h\right)=(2,0,0.5)$ and different velocities, $v=0.51,1.0$ and 1.5. The different 'velocity rays' are indexed: 1 - vertical ray, 2 - source ray, 3 - isochron-normal ray, and 4 - canonical ray (continuation characteristic). 
2. Iversen [12]: source-'ray' parametrization. Iversen defines a 'velocity ray' as the curve connecting an initial with a perturbed isochron, subject to the condition

$$
\begin{array}{r}
{\left[\partial_{\alpha}\left(v[\alpha](s, 0) \partial_{s} t_{s}(\alpha ; s, x, z)\right)+v[\alpha](s, 0) \frac{\mathrm{d}(x, z)}{\mathrm{d} \alpha} \cdot \partial_{(x, z)} \partial_{s} t_{s}(\alpha ; s, x, z)\right]_{s=y^{\prime}-h}=0,} \\
(x, z)=(x, z)(\alpha) .
\end{array}
$$

(This equation arises from the composition-like relation

$$
\left.v[\alpha](s, 0) \partial_{s} t_{s}(\alpha ; s, x, z)-v\left[\alpha_{0}\right](s, 0) \partial_{s} t_{s}\left(\alpha_{0} ; s, x_{0}, z_{0}\right)=0, \quad s=y^{\prime}-h .\right)
$$

For the constant velocity models, with $t_{s}$ as in (56), we thus obtain the supplementary equation

$$
\frac{\mathrm{d} x}{\mathrm{~d} v}-\tan \beta \frac{\mathrm{d} z}{\mathrm{~d} v}=0, \quad \tan \beta=\frac{x-y^{\prime}+h}{z} .
$$

Equations (57) and (66) can be solved for $\frac{\mathrm{d}(x, z)}{\mathrm{d} v}$; the corresponding curves are illustrated, and indexed by 2, in Fig. 4.

3. Meng and Bleistein [20]: isochron-normal 'ray'. The authors define a 'velocity ray' as the curve connecting an initial with a perturbed isochron, with the provision that the curve is normal to the (initial) isochron:

$$
\partial_{(x, z)} T\left(\alpha ; y^{\prime}, x, z\right) \wedge \frac{\mathrm{d}(x, z)}{\mathrm{d} \alpha}=0 .
$$

We introduce the isochron-normal vector, $n=\partial_{(x, z)} T /\left\|\partial_{(x, z)} T\right\|$, and half of opening angle between incident and reflected rays, $\theta$ (Fig. 1), so that $\left\|\partial_{(x, z)} T\right\|=\frac{2 \cos \theta}{v[\alpha](x, z)}$. From equality and equation (63) we get that

$$
n \cdot \frac{\mathrm{d}(x, z)}{\mathrm{d} \alpha}=-\partial_{\alpha} T \frac{v[\alpha]}{2 \cos \theta}
$$

$[19,(4.4 .24),(4.4 .25)]$ is the velocity of an isochron-normal 'ray'.

For the constant velocity models, with $T$ as in (50), (56), we get

$$
\partial_{z} T\left(v ; y^{\prime}, x, z\right) \frac{\mathrm{d} x}{\mathrm{~d} v}-\partial_{x} T\left(v ; y^{\prime}, x, z\right) \frac{\mathrm{d} z}{\mathrm{~d} v}=0 .
$$

Equations (57) and (69) can be solved for $\frac{\mathrm{d}(x, z)}{\mathrm{d} v}$; the corresponding curves (isochron-normal 'rays') are illustrated, and indexed by 3 , in Fig. 4 .

4. Adler [14], Iversen [12] (combined parametrization): canonical 'ray'. Adler honors all components of equation (51); in the constant background media case equation

$$
-\frac{\partial_{y^{\prime}} \rho_{s}+\partial_{y^{\prime}} \rho_{r}}{v^{2}}+\frac{\partial_{x} \partial_{y^{\prime}} \rho_{s}+\partial_{x} \partial_{y^{\prime}} \rho_{r}}{v} \frac{\mathrm{d} x}{\mathrm{~d} v}+\frac{\partial_{z} \partial_{y^{\prime}} \rho_{s}+\partial_{z} \partial_{y^{\prime}} \rho_{r}}{v} \frac{\mathrm{d} z}{\mathrm{~d} v}=0
$$


is supplementary to (57). Equations (57) and (70) can be solved for $\frac{\mathrm{d}(x, z)}{\mathrm{d} v}$; the corresponding curves are illustrated, and indexed by 4 , in Fig. 4 . They can be related to the so-called combinedray parametrization of velocity rays in [12,13]; see also the Appendices in [14].

Remark. We verify which of the velocity 'rays' are actually rays. First we note that a set of continuation 'lines' is implicitly defining a vector field $V_{\alpha}$ that, in turn, defines a continuation Hamiltonian if the respective Lie derivative $\mathcal{L}_{V_{\alpha}} \omega$ is zero (see discussion at the end of Section 4.1). Thus checking integrability conditions (35) allows making conclusion about existence of the Hamiltonian. The construction above led to expressions for $\frac{\mathrm{d}(x, z)}{\mathrm{d} v}$ in terms of coordinates $\left(x, z, \tau, y^{\prime}\right)$. Here, we introduce a procedure to, consistently, construct $\frac{\mathrm{d}(\xi, \zeta)}{\mathrm{d} v}$.

First we notice that equations (59) can be solved explicitly providing $\tau=\tau(\alpha ; x, z, \xi, \zeta), y^{\prime}=$ $y^{\prime}(\alpha ; x, z, \xi, \zeta)$ (expression for $y^{\prime}$ is given in (60)). Then we can write:

$$
V_{1}(\alpha ; x, z, \xi, \zeta)=\frac{\mathrm{d}(x, z)}{\mathrm{d} \alpha}=f\left(\alpha ; x, z, y^{\prime}(\alpha ; x, z, \xi, \zeta)\right)
$$

where $f\left(\alpha ; x, y^{\prime}\right)$ will take different forms for the different velocity 'rays' introduced above.

We note that equations (59) (or (54)) must hold along the 'ray' $(x(\alpha), z(\alpha))$, providing $(\xi(\alpha), \zeta(\alpha))=$ $\tau \partial_{(x, z)} T\left(\alpha ; y^{\prime}, x(\alpha), z(\alpha)\right)$. Further differentiating it with respect to $\alpha$ (keeping $\tau$ and $y^{\prime}$ fixed):

$$
V_{2}(\alpha ; x, z, \xi, \zeta)=\frac{\mathrm{d}(\xi, \zeta)}{\mathrm{d} \alpha}=g\left(\alpha ; x, z, \tau(\alpha ; x, z, \xi, \zeta), y^{\prime}(\alpha ; x, z, \xi, \zeta)\right),
$$

where $g\left(\alpha ; x, z, \tau, y^{\prime}\right)=\tau \partial_{\alpha} \partial_{(x, z)} T\left(\alpha ; y^{\prime}, x, z\right)+\tau \frac{\mathrm{d}(x, z)}{\mathrm{d} \alpha} \cdot \partial_{(x, z)} \partial_{(x, z)} T\left(\alpha ; y^{\prime}, x, z\right)$.

With expressions for $\left(V_{1}, V_{2}\right)$, obtained in (71) and (72), we can test conditions (35): all 'rays' except for the canonical ray (case 4) fail to pass this test. Only velocity 'rays' corresponding with canonical transformations yield the appropriate geometry underlying an evolution-equation based approach to image continuation. We argue that, hence, velocity 'rays' other than the canonical rays (continuation characteristics) should perhaps not be called rays.

\section{Velocity continuation of common-image point gathers in the presence of caustics}

In this section, we illustrate, numerically, method 1 (Section 4.1 ) by computing $\Lambda_{\left(\alpha, \alpha_{0}\right)}$ and $P(\alpha)$. We consider the problem of velocity continuation of so-called common-image point gathers in the presence of caustics. The formation of such gathers is summarized in Appendix B; the analysis of the underlying angle transform, $\mathcal{A}_{\text {we }}$, can be found in [37]. We write

$$
\mathcal{A}_{w e}(\alpha): u(s, r, t) \rightarrow w_{g}(\alpha ; x, z, p)
$$


if $u$ represents the data and $w_{g}$ the common-image point gathers. For purpose of this example one can think of operator $\mathcal{A}_{w e}$ as a 'black box' for which we can calculate a canonical relation $\Lambda_{\mathcal{A}_{w e}} ; w_{g}$ is closely related angle gathers; $p$ is related to scattering angle in the beam-forming approach (cf. [47]) or subsurface offset in the differential semblance approach (cf. [34]). The operators $\mathcal{A}_{w e}(\alpha)$ are microlocally invertible under certain conditions on the ray geometry [48] (for small $p$ ). The general application of the continuation theory developed here to the downward continuation approach and associated angle transform [32,33] can be found in [49].
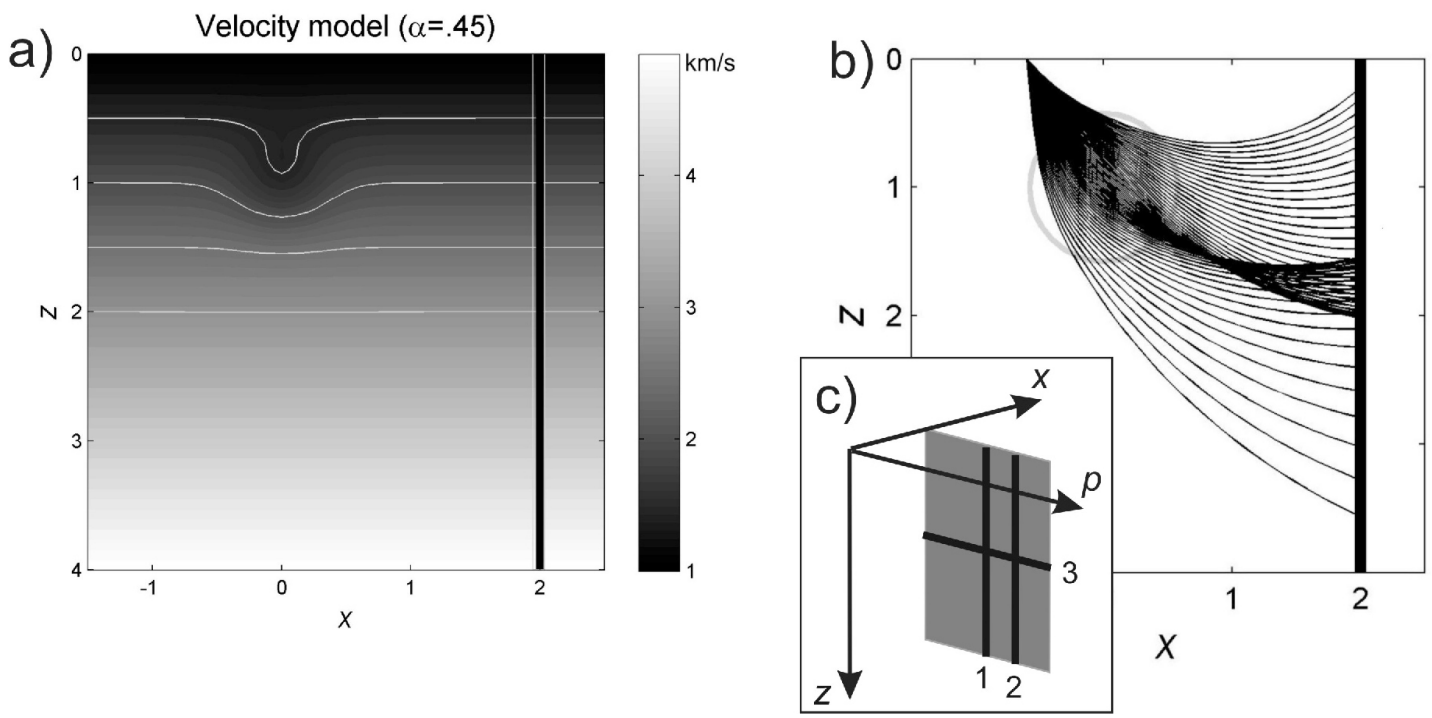

Fig. 5. (a) - Background velocity model shown in color (linear gradient and a low velocity lens), and vertical reflector $x=2$. The gray circle indicates the location of the lens; (b) - incident rays for a single point source; (c) - a schematic view of $(x, z, p)$-space on which $w_{g}(x, z, p)$ is defined; the singular support of the correct image - corresponding with the vertical reflector - is indicated by a gray plane.

As the family of background models $v[\alpha]$, we take

$$
v[\alpha]=1+z-\alpha \exp \left[-7.5\left(x^{2}+(1-z)^{2}\right)\right],
$$

containing a vertical gradient and a low velocity lens, where $\alpha$ defines the 'strength' of the lens. We take $\alpha_{0}=0.45$ as the true model (see Fig. 5 a) and construct a reflected wave traveltime surface (singularities in data $u$ will be supported on this surface) for a vertical reflector at $x=2$ (thick vertical line in Fig. 5 a) which can be thought of as a toy model for a salt dome flank). In Fig. 5 b) we show incident rays for a single point source. We observe the presence of caustics and turning rays. The singular support of $w_{g}\left(\alpha_{0} ; x, z, p\right)$ (for the true model) is sketched in Fig. 5 c). The true position of vertical reflector is replicated in $p$ direction composing a plane (in gray).

We apply continuation operator $C_{\left(\alpha, \alpha_{0}\right)}^{\mathcal{A}}=\mathcal{A}_{w e}(\alpha) \mathcal{A}_{w e}^{-1}\left(\alpha_{0}\right)$, while numerically composing the canonical relations, $\Lambda_{\mathcal{A}_{w e}}(\alpha) \circ \Lambda_{\mathcal{A}_{w e}}^{-1}\left(\alpha_{0}\right)$, to $w_{g}\left(\alpha_{0} ; x, z, p\right)$. We illustrate the continuation and associated characteristics by flowing out the three lines depicted in Fig. 5 c). Fig. 6 shows the 

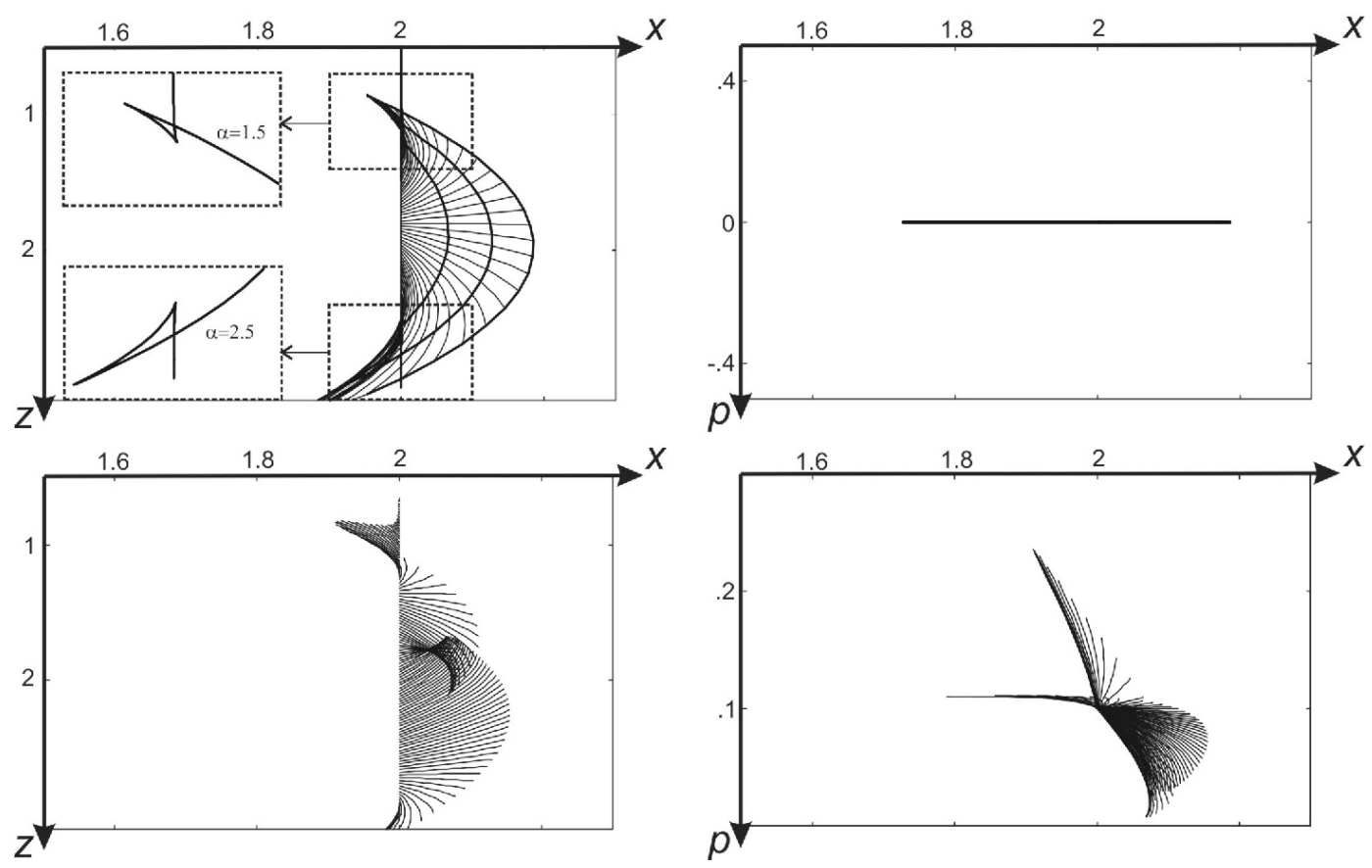

Fig. 6. Image continuation, $w_{g}(x=2, z, p=0)$ (top) and $w_{g}(x=2, z, p=0.1)$ (bottom). The continuation characteristics are projected onto the $(x, z)$ plane (left) and the $(x, p)$ plane (right). In the top left figure we also plotted four fronts (thick solid lines), at $\alpha=\alpha_{0}=0.45$ (true model), $\alpha=0.35$, $\alpha=0.25$ and $\alpha=0.15$. The inserts show the cusps at the top and the bottom in more detail; these cusps are formed in a transitional region, where the influence of the lens vanishes.

evolution of lines 1 and 2, both representative of the vertical reflector at different values of $p$. Note that, for $p=0$, the continuation characteristics stay in a plane, unlike for $p=0.1$. Fig. 7 shows the evolution of line 3, a 'string' in a common-image point gather, in Fig. $5 \mathrm{c}$ ). In Fig. 8 a) we illustrate the computed principal symbol $p_{1}\left(\alpha, x, z, p, \xi_{x}, \xi_{z}, \xi_{p}\right)$ in the form of a phase velocity surface that is a level-set surface $p_{1}\left(\alpha, x, z, p, k_{x}, k_{z}, k_{p}\right)^{-1}=1$ for fixed $(\alpha, x, z, p)=(0.45,1,1,0)$, where $k_{x}=\xi_{x} / \xi_{\alpha}, k_{z}=\xi_{z} / \xi_{\alpha}, k_{p}=\xi_{p} / \xi_{\alpha}$. Solving this equation we get a phase velocity surface $v_{p h}\left(k_{x}, k_{z}, k_{p}\right)$ that it is not a closed surface as not for all directions $\left(k_{x}, k_{z}, k_{p}\right)$ operator $\mathcal{A}_{w e}$ is determined. Fig. $8 \mathrm{~b}$ ) shows part of the phase velocity surface corresponding to small subsurface offsets (region where invertibility of $\mathcal{A}_{w e}$ was proven in [37]).

\section{Discussion}

We developed the foundation of, and a comprehensive framework for seismic continuation, while extending the earlier approaches to this type of continuation to allow for the formation of caustics. We illustrated how the concepts introduced and developed by Fomel (partial differen- 
tial equations for data and image continuation, corresponding Hamiltonians), Goldin (continuation by composing remigration with demigration, underlying contact transformations), Hubral et al. (image waves), Iversen (system of ordinary differential equations for continuation characteristics, connection with ray perturbation theory), Adler (velocity rays, connection with a migration Jacobian) and Bleistein et al. (common-offset image continuation and residual moveout) are contained in our theory.
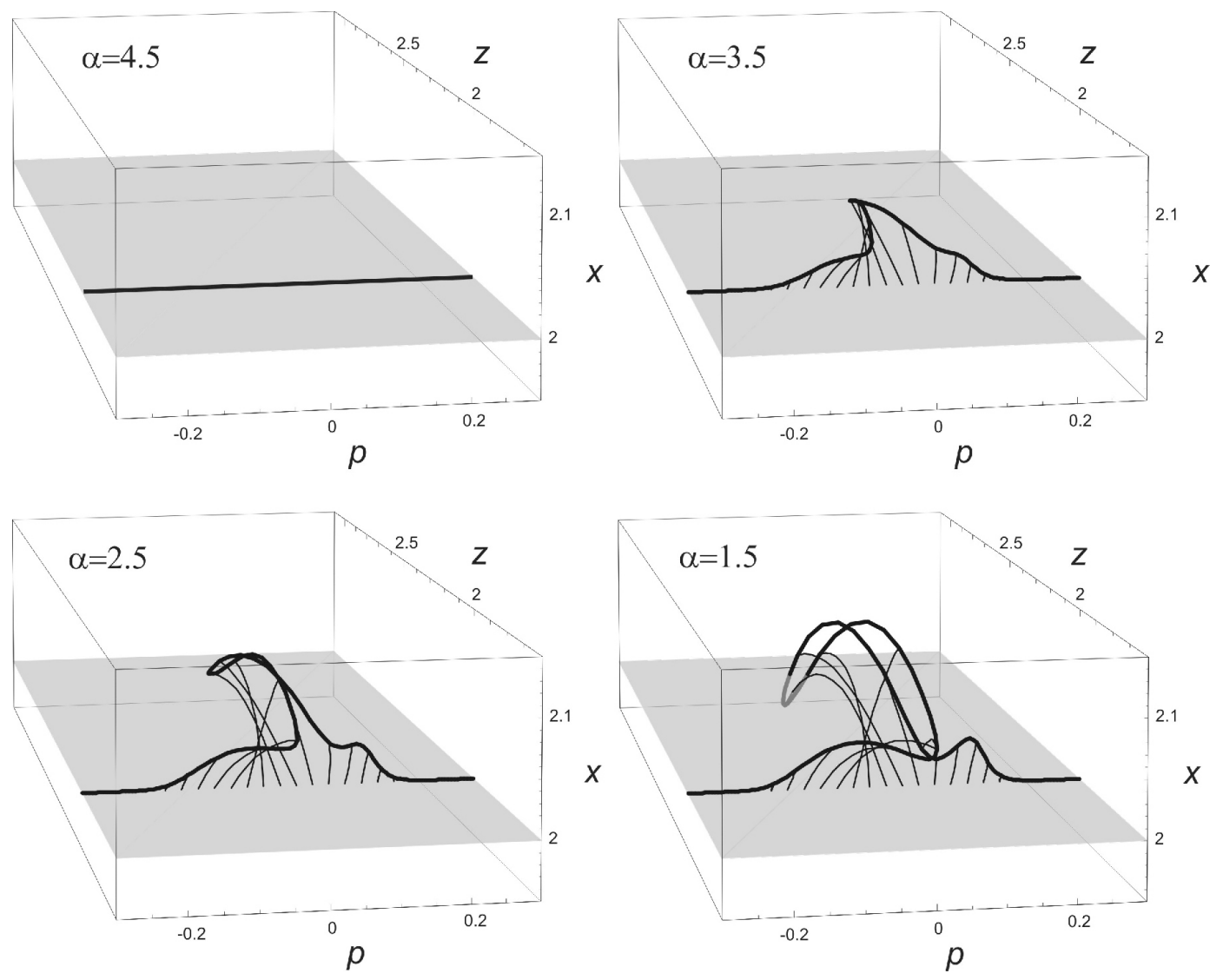

Fig. 7. Continuation of a line or 'string' (bold line), initially at $z=2$, in a 'vertical' common-image point gather initially at $x=2$ (line 3 in Fig. 5 c). The thin lines indicate continuation characteristics.

In our analysis we formulate conditions on the originating family of operators under which continuation is properly defined. We showed the existence and provided the construction of a hyperbolic evolution equation that describes the process of continuation. As a byproduct, we obtained a global Hamiltonian, the flow of which determines the propagation of singularities under continuation. In, for example, Iversen's work such a Hamiltonian was not identified. The symbol of the evolution equation will, in general, have to be determined numerically. Computationally, the advantage is avoiding the remigration of all the data, the relative saving being comparable to map migration versus imaging. A particular promising strategy to solve the evolution equation can be found in [50] leading to 'compressive' continuation. It is based on a sparse approximation of the initial data in terms of sums of curvelets, while the procedure is 

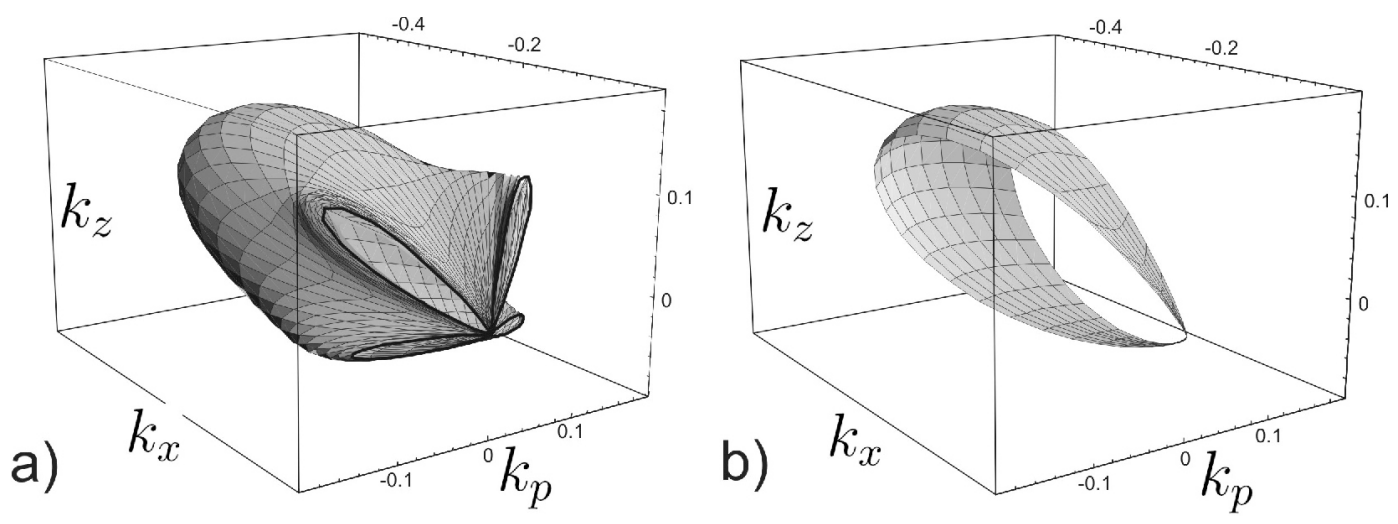

Fig. 8. Phase velocity surface for common-image point gather velocity continuation operator; (a) - whole surface; (b) - part corresponding to small-offsets (where operator $\mathcal{A}_{w e}$ is proven to be invertible).

initiated by an approximate solution that subjects the curvelet components to a rigid motion in accordance with the continuation Hamilton flow.

Velocity continuation has applications, for example, in wave-equation reflection tomography [51]. (The measure whether a background model (the value of $\alpha$ ) is acceptable for imaging, essentially, depends on flatness of the common-image point gathers (illustrated in previous section) in $p$ direction.) In this context, the evolution operator associated with image continuation with velocity aids in the evaluation of the so-called sensitivity kernels. As reflected by the tomographic sensitivity kernels, continuation senses the velocity model in an integrated fashion.

\section{Acknowledgements}

$\mathrm{MdH}$ has fond memories of the many discussions with Sergey Goldin that led to the research presented in this paper. The authors thank an unknown referee for her/his comments that helped to improve the paper. This research was supported in part by the members, BP, ConocoPhillips, ExxonMobil, StatoilHydro and Total, of the Geo-Mathematical Imaging Group.

\section{A Oscillatory integral representations - change of phase variables}

In applications, the oscillatory integral representation of the relevant operator kernel often appears naturally in a form different from the canonical form (12)-(13). We set $n_{Y}=n_{X}=n$. If for the kernel of an FIO in $\mathcal{C}$ we have an oscillatory integral representation - making use of coordinates $\left(y, x_{I}, \xi_{J}\right)$ with $I \cup J=\{1, \ldots, n\}$ on $\Lambda$ - with amplitude $a=a^{\prime}\left(y, x_{I}, \xi_{J}\right)$, we can 
obtain $a=a(y, \xi)$ by the relation $[52,4.1 .2]^{5}$

$$
a(y, \xi) \exp [\mathrm{i} S(y, \xi)]=\int a^{\prime}\left(y, x_{I}^{\prime}, \xi_{J}\right) \exp \left[\mathrm{i}\left(S^{\prime}\left(y, x_{I}^{\prime}, \xi_{J}\right)+\left\langle\xi_{I}, x_{I}^{\prime}\right\rangle\right)\right] \mathrm{d} x_{I}^{\prime},
$$

which follows from writing the action of the associated FIO as

$$
\begin{aligned}
(F u)(y)=\int(2 \pi)^{-n} \iiint a^{\prime}\left(y, x_{I}^{\prime}, \xi_{J}\right) \exp \left[\mathrm{i}\left(S^{\prime}\left(y, x_{I}^{\prime}, \xi_{J}\right)+\left\langle\xi_{I}, x_{I}^{\prime}\right\rangle\right)\right] \mathrm{d} x_{I}^{\prime} \\
\exp \left[\mathrm{i}\left(-\left\langle\xi_{J}, x_{J}\right\rangle-\left\langle\xi_{I}, x_{I}\right\rangle\right)\right] \mathrm{d} \xi_{J} \mathrm{~d} \xi_{I} u(x) \mathrm{d} x
\end{aligned}
$$

Invoking the method of stationary phase in $x_{I}$ yields

$$
\begin{array}{r}
\int a^{\prime}\left(y, x_{I}, \xi_{J}\right) \exp \left[\mathrm{i}\left(S^{\prime}\left(y, x_{I}, \xi_{J}\right)+\left\langle\xi_{I}, x_{I}\right\rangle\right)\right] \mathrm{d} x_{I} \\
=(2 \pi)^{|I| / 2} \exp \left[\mathrm{i}(\pi / 4) \operatorname{sgn} \Delta\left(y, x_{I}, \xi_{J}\right)\right] a^{\prime}\left(y, x_{I}, \xi_{J}\right) \\
\exp \left[\left.\mathrm{i}\left(S^{\prime}\left(y, x_{I}, \xi_{J}\right)\right] \exp \left[\mathrm{i}\left\langle\xi_{I}, x_{I}\right\rangle\right]\left[\operatorname{det} \Delta\left(y, x_{I}, \xi_{J}\right)\right]^{-1 / 2}\right|_{x_{I}=x_{I}(y, \xi)},\right.
\end{array}
$$

where $\Delta$ is the $|I| \times|I|$ Hessian

$$
\Delta\left(y, x_{I}, \xi_{J}\right)=\frac{\partial^{2} S^{\prime}\left(y, x_{I}, \xi_{J}\right)}{\partial x_{I}^{2}} .
$$

We identify $S(y, \xi)=\left.\left(S^{\prime}\left(y, x_{I}, \xi_{J}\right)+\left\langle\xi_{I}, x_{I}\right\rangle\right)\right|_{x_{I}=x_{I}(y, \xi)}$. The stationary points are the $x_{I}$, satisfying the system of equations

$$
-\frac{\partial S^{\prime}}{\partial x_{I}}\left(y, x_{I}, \xi_{J}\right)=\xi_{I}
$$

with solution $x_{I}=x_{I}\left(y, \xi_{I}, \xi_{J}\right)=x_{I}(y, \xi)$ revealing the coordinate transformation $\left(y, x_{I}, \xi_{J}\right)$ $\rightarrow\left(y, \xi_{I}, \xi_{J}\right)$ on $\Lambda$. We note that

$$
(-)^{|I|} \operatorname{det} \frac{\partial^{2} S^{\prime}\left(y, x_{I}, \xi_{J}\right)}{\partial x_{I}^{2}}=\operatorname{det} \frac{\partial\left(\xi_{I}\right)}{\partial\left(x_{I}\right)} .
$$

\section{B Wave-equation angle transform}

In the presence of caustics, the framework of common-offset migration no longer applies, and we resort to an alternative invertible transformation. With data $u=u(s, r, t)$ (identifying $(s, r, t)$

$\overline{5}$ Follows by inserting the Fourier transforms $\mathcal{F}_{\xi_{I} \rightarrow x_{I}}^{-1} \mathcal{F}_{x_{I} \rightarrow \xi_{I}}$ in front of $u(x)$ in the action of $F$ on $u$. 
as coordinates for $y$ ), common-image point gathers are now formed as follows. We will have $n_{X}=n_{Y}=3$ for two-dimensional configurations. Let $G$ denote the causal Green's function of the scalar wave equation. We then introduce $[36,37,51]$

$$
\begin{aligned}
& \mathrm{D}\left(x-h_{x}, x+h_{x}, z-h_{z}, z+h_{z}, t^{\prime}\right)= \\
& \iiint \int G\left(x+h_{x}, z+h_{z},-(\widetilde{t}-t), r, 0\right) G\left(x-h_{x}, z-h_{z}, \widetilde{t}-t^{\prime}, s, 0\right) \mathrm{d} \widetilde{t} \partial_{t}^{2} u(s, r, t) \mathrm{d} r \mathrm{~d} s \mathrm{~d} t .
\end{aligned}
$$

We have the freedom of choosing the direction of $\left(h_{x}, h_{z}\right)$ [53]. For the case of non-horizontal wave propagation and non-vertical reflectors, a natural choice is $h_{z}=0$ (leading to the downward continuation approach to imaging [37]). In the case of near vertical reflectors, we choose $h_{x}=0$. We then form an image gather according to

$$
w_{g}(x, z, p)=\int \mathrm{D}\left(x, x, z-h_{z}, z+h_{z}, 2 p h_{z}\right) \chi\left(x, z, h_{z}\right) \mathrm{d} h_{z},
$$

where $p$ is a variable related to the scattering (opening) angle at point $(x, z)$, and $\chi\left(x, z, h_{z}\right)$ is a cutoff in $h_{z}$. (This type of transform was introduced in [47].) Equations (B.1)-(B.2) define the so-called angle transform [36,37], $\mathcal{A}_{w e}: u(s, r, t) \rightarrow w_{g}(x, z, p)$. It can be shown that the operator $\mathcal{A}_{w e}$ is microlocally invertible for the velocity models and vertical reflector considered here [48].

\section{References}

[1] S. Fomel, Kinematically equivalent differential operator for offset continuation of seismic sections, Russian Geology and Geophysics 35, No. 9 (1994) 122134.

[2] M. De Hoop, G. Uhlmann, Characterization and 'source-receiver' continuation of seismic reflection data, Comm. Math. Phys. 263 (2006) 1-19.

[3] S. Fomel, Method of velocity continuation in the problem of seismic time migration, Russian Geology and Geophysics 35, No. 5 (1994) 100-111.

[4] S. Goldin, Theoretical aspects of 3D-DMO, 72nd Annual International Meeting: Expanded Abstracts, SEG (2002) 2333-2336.

[5] S. Fomel, Theory of differential offset continuation, Geophysics 68 (2003) 718-732.

[6] J. Etgen, Residual prestack migration and interval velocity estimation, Ph.D. thesis, Stanford University (1990).

[7] R. Stolt, Short note - A prestack residual time migration operator, Geophysics 61 (1996) 605-607.

[8] P. Sava, Prestack residual migration in the frequency domain, Geophysics 68 (2003) 634-640. 
[9] M. Tygel, J. Schleicher, P. Hubral, A unified approach to 3-D seismic reflection imaging, Part II: Theory, Geophysics 61 (1996) 759775.

[10] P. Hubral, M. Tygel, J. Schleicher, Seismic image waves, Geophys. J. Int. 125 (1996) 431-442.

[11] S. Fomel, Velocity continuation and the anatomy of residual prestack time migration, Geophysics 68 (2003) 1650-1661.

[12] E. Iversen, Derivatives of reflection point coordinates with respect to model parameters, Pure and Appl. Geoph. 148 (1996) 287-317.

[13] E. Iversen, Velocity rays for heterogeneous anisotropic media: Theory and implementation, Geophysics 71 (2006) T117-T127.

[14] F. Adler, Kirchhoff image propagation, Geophysics 67 (2002) 126-134.

[15] E. Iversen, The isochron ray in seismic modeling and imaging, Geophysics 69 (2004) 1053-1070.

[16] T. Alkhalifah, M. De Hoop, Integral DMO in anisotropic media, 66th Annual Meeting: Expanded Abstracts, Denver, SEG (1996) 491-494.

[17] T. Alkhalifah, M. De Hoop, Residual dip moveout in VTI media, Geophys. Prosp. 53 (2005) 1-12.

[18] Z. Liu, N. Bleistein, Migration velocity analysis: Theory and an iterative algorithm, Geophysics 60 (1995) 142-153.

[19] Z. Meng, Tetrahedral based earth models, ray tracing in tetrahedral models and analytical migration velocity analysis, Ph.D. thesis, Colorado School of Mines (1999).

[20] Z. Meng, N. Bleistein, On velocity/depth ambiguity in 3-D migration velocity analysis (short note), Geophysics 66 (2001) 256-260.

[21] S. Goldin, Superposition and continuation of operators used in seismic imaging, Russian Geology and Geophysics No. 9 (1994) 131-145.

[22] S. Goldin, Geometric fundamentals of seismic imaging: A geometric theory of the upper level, in: Amplitude-preserving seismic reflection imaging, Proceedings of the Workshop, Geopysical Press, London, 1998, pp. 120-223.

[23] S. Goldin, Geometrical fundamentals of seismic imaging: realization of contact mappings, Siberian J. of Numer. Mathematics 6(4) (2003) 323-345.

[24] J. Duistermaat, Fourier Integral Operators, Birkhäuser, New York, 1996.

[25] F. Treves, Introduction to Pseudodifferential and Fourier Integral Operators, Vol. 2, Plenum Press, New York, 1980.

[26] L. Hörmander, The Analysis of Linear Partial Differential Operators, Vol. 3, Springer-Verlag, Berlin, 1985. 
[27] L. Hörmander, The Analysis of Linear Partial Differential Operators, Vol. 4, Springer-Verlag, Berlin, 1985.

[28] G. Beylkin, Imaging of discontinuities in the inverse scattering problem by inversion of a causal generalized Radon transform, J. Math. Phys. 26 (1985) 99-108.

[29] Rakesh, A linearized inverse problem for the wave equation, Comm. Partial Differential Equations 13 (1988) 573-601.

[30] A. Ten Kroode, D. Smit, A. Verdel, A microlocal analysis of migration, Wave Motion 28 (1998) $149-172$.

[31] M. De Hoop, S. Brandsberg-Dahl, Maslov asymptotic extension of generalized Radon transform inversion in anisotropic elastic media: A least-squares approach, Inverse Problems 16 (2000) 519562.

[32] C. Stolk, M. De Hoop, Microlocal analysis of seismic inverse scattering in anisotropic, elastic media, Comm. Pure Appl. Math. 55 (2002) 261-301.

[33] C. Stolk, M. De Hoop, Modeling of seismic data in the downward continuation approach, SIAM J. Appl. Math. 65 (2005) 1388-1406.

[34] W. Symes, J. Carazzone, Velocity inversion by differential semblance optimization, Geophysics 56 (1991) 654-663.

[35] C. Stolk, W. Symes, Smooth objective functionals for seismic velocity inversion, Inverse Problems 19 (2003) 73-89.

[36] C. Stolk, M. De Hoop, Seismic inverse scattering in the 'wave-equation' approach, MSRI preprint \#2001-047.

[37] C. Stolk, M. De Hoop, Seismic inverse scattering in the downward continuation approach, Wave Motion 43 (2006) 579-598.

[38] J. Duistermaat, V. W. Guillemin, The spectrum of positive elliptic operators and periodic bicharacteristics, Inventiones Mathematicae 29 (1975) 39-79.

[39] H. Omori, Infinite-dimensional Lie groups, Amer. Math. Soc., Providence, 1997.

[40] R. Schmid, Infinite dimensional lie groups with applications to mathematical physics, Journal of Geometry and Symmetry in Physics 1 (2004) 1-67.

[41] J. Duistermaat, L. Hörmander, Fourier integral operators II, Acta Mathematica 128 (1972) 184-269.

[42] A. Weinstein, Lectures on symplectic manifolds, Vol. 29, Regional Conference Series in Mathematics, American Mathematical Society, Providence, RI, 1977.

[43] F. Warner, Foundations of differentiable manifolds and Lie groups, Graduate Texts in Mathematics, vol. 94, Springer-Verlag, 1983. 
[44] N. Bleistein, J. Cohen, J. Stockwell, Mathematics of multidimensional seismic imgaing, migration, and inversion, Springer-Verlag, New York, 2001.

[45] H. Douma, M. De Hoop, Leading-order seismic imaging using curvelets, Geophysics 72 (2007) S231-S248.

[46] A. Duchkov, M. de Hoop, S. Fomel, Continuation of a class of seismic processors and associated 'rays', 76th Annual Meeting: Expanded Abstracts, New Orleans, SEG (2006) 2549-2553.

[47] C. De Bruin, C. Wapenaar, A. Berkhout, Angle-dependent reflectivity by means of prestack migration, Geoph. 55 (1990) 1223-1234.

[48] C. Stolk, M. De Hoop, Curvilinear wave-equation angle transform: Caustics, turning rays, absence of kinematic artifacts, 77th Annual Meeting: Expanded Abstracts, San Antonio, SEG (2007) 21802184.

[49] A. Duchkov, M. De Hoop, Velocity continuation in the downward continuation approach to seismic imaging, Geophys. J. Int. submitted.

[50] F. Andersson, M. De Hoop, H. Smith, G. Uhlmann, A multi-scale approach to hyperbolic evolution equations with limited smoothness, Comm. Partial Differential Equations in print.

[51] M. De Hoop, R. Van der Hilst, P. Shen, Wave-equation reflection tomography: Annihilators and sensitivity kernels, Geoph. J. Int. 167 (2006) 1332-1352.

[52] A. Mishchenko, V. Shatalov, B. Sternin, Lagrangian manifolds and the Maslov operator, SpringerVerlag, Berlin, 1990.

[53] C. Stolk, M. De Hoop, W. Symes, Kinematics of shot-geophone migration, Geophysics submitted. 